\title{
On the Fundamental Constants of Nature
}

\section{David Humpherys}

david.humpherys.wg00@wharton.upenn.edu

https://orcid.org/0000-0001-7375-7897

\begin{abstract}
Planck units of length, mass, and time are fundamental constants of nature. Traditional constants including Planck's constant, the gravitational constant, the elementary charge, and many others are comprised of these three fundamental units. Physics equations are functions in which maximum potentials defined by the Planck units are reduced by one or more proportionality operators, producing observed quantities of natural phenomena. Natural symmetries constrain the relationships between length, mass, and time, yielding the physical dynamics of momentum, action, force, and energy. The Planck units quantify mechanical, gravitational, and electromagnetic properties of the universe and offer a common language for interpreting the standard model interactions. Units associated with the electromagnetic interaction are translated into units of length, mass, and time, including the coulomb, ampere, volt, tesla, henry, weber, farad, ohm, and siemen.
\end{abstract}

Keywords Planck units · natural units · fundamental constants · physical constants · Planck's constant $\cdot$ gravitational constant $\cdot$ particle mechanics

\section{Introduction}

At the close of the 19th century, Max Planck unveiled the constant of proportionality that bears his name today [1-3]. Planck's discovery ushered in a new era of quantum physics and $\hbar$ became ubiquitous in equations describing the physical universe on small scales. At the same time, Planck showed that combining $\hbar, G$, and $c$ in the right proportions creates natural quantities of length, mass, and time.

More than a century later, the system of derived Planck units-featuring enigmatic ratios of the same three constants-has expanded deeper into the fields of quantum mechanics, thermodynamics, electromagnetism, and gravity [4-9]. Despite sharing the same building blocks, these formulas offer little insight into the physical meaning of $\hbar$ and $G$, or the Planck units they define.

A deeper examination of the physical constants and the entire system of Planck units reveals a beautifully simple structure that warrants re-evaluation of what is fundamental. The opportunity eluding Planck at the time, and many others since, is recognizing that the 
fundamental ingredients of the universe are measured in units of length, mass, and timenot in quantities of Planck's constant or the Gravitational constant. From the relationships between these three fundamental units emerge the symmetries on which modern day physical theories are built. The fundamental Planck units not only explain the entire system of Planck units more simply and elegantly; they redefine the most important physical constants and equations in ways that expand our understanding of the natural universe.

Planck's constant and the gravitational constant can be stated in Planck units as

$$
\begin{aligned}
\hbar & =l_{P} m_{P} c \\
G & =\frac{l_{P}}{m_{P}} c^{2} .
\end{aligned}
$$

Similarities between the two constants are immediately apparent in these simple forms, most notably in the relationship between length and mass. The product form of the relationship found in Planck's constant is ideal for determining the extensive properties of elementary particles, whereas the quotient form of the gravitational constant is better suited for calculating the intensive properties of large gravitational bodies.

Equations 1 and 2 can be derived from the equations of Planck length and Planck mass, shown in appendix A. The composition of Planck's constant and the gravitational constant are demonstrated in table 1 using CODATA values.

Table 1: Planck's constant and the gravitational constant are comprised of fundamental units of length, mass, and time.

\begin{tabular}{lrrl}
\hline Constant & & Unit & Value \\
\hline$\hbar=$ & & $l_{P}$ & $1.616255 \times 10^{-35} \mathrm{~m}$ \\
& $\times$ & $m_{P}$ & $2.176434 \times 10^{-8} \mathrm{~kg}$ \\
& $\times$ & $c$ & $299,792,458 \mathrm{~m} / \mathrm{s}$ \\
& $=$ & & $\mathbf{1 . 0 5 4 5 7 2 \times 1 0 ^ { - 3 4 }} \mathbf{k g m}^{\mathbf{2}} / \mathrm{s}^{\mathbf{2}}$ \\
& & & \\
$G=$ & & $l_{P}$ & $1.616255 \times 10^{-35} \mathrm{~m}$ \\
& $\div$ & $m_{P}$ & $2.176434 \times 10^{-8} \mathrm{~kg}$ \\
& $\times$ & $c$ & $299,792,458 \mathrm{~m} / \mathrm{s}$ \\
$\times$ & $c$ & $299,792,458 \mathrm{~m} / \mathrm{s}$ \\
& $=$ & & $\mathbf{6 . 6 7 4 3 0 \times 1 0 ^ { - 1 1 }} \mathbf{m}^{\mathbf{3}} / \mathbf{k g s}^{\mathbf{2}}$ \\
\hline
\end{tabular}

The knowledge that $\hbar$ and $G$ are comprised of more fundamental quantities is a basis for reinterpreting physical laws explained by these two constants. For all the equations that rely on Planck's constant and the gravitational constant, it is in the relationships between a handful of Planck units that we obtain definitive predictions about the world.

Table 2 gives an extensive list of physical constants restated in fundamental quantities of length, mass, and time. Each constant is defined in elementary form using the three fundamental Planck units, and additional forms are given in units of Planck energy, Planck momentum, and the speed of light. 
Table 2: Traditional constants are composites of more fundamental quantities of length, mass, and time. The elementary form of these constants reveals physical meaning that was previously obfuscated by the composite values.

\begin{tabular}{|c|c|c|c|c|c|c|}
\hline Constant & Sym & Std form & Elem form & Alt forms & MKS value & EM value \\
\hline Planck length & $l_{p}$ & $\sqrt{\frac{\hbar G}{c^{3}}}[1]$ & $l_{p}$ & - & $1.616255 \times 10^{-35} \mathrm{~m}$ & - \\
\hline Planck mass & $m_{p}$ & $\sqrt{\frac{\hbar c}{G}}[1]$ & $m_{p}$ & - & $2.176434 \times 10^{-8} \mathrm{~kg}$ & - \\
\hline Planck time & $t_{p}$ & $\sqrt{\frac{\hbar G}{c^{5}}}[1]$ & $t_{p}$ & - & $5.391247 \times 10^{-44} s$ & - \\
\hline Planck momentum & $p_{p}$ & $\sqrt{\frac{\hbar c^{3}}{G}}[4,5]$ & $\frac{l_{p} m_{p}}{t_{p}}$ & $m_{p} c$ & $6.524784 \frac{\mathrm{kgm}}{\mathrm{s}}$ & - \\
\hline Planck energy & $E_{p}$ & $\sqrt{\frac{\hbar c^{5}}{G}}[4,9]$ & $\frac{l_{p}^{2} m_{p}}{t_{p}^{2}}$ & $m_{p} c^{2}$ & $1,956,081,000 \frac{\mathrm{kgm}^{2}}{\mathrm{~s}^{2}}$ & - \\
\hline Reduced Planck const & $\hbar$ & - & $\frac{l_{p}^{2} m_{p}}{t_{p}}$ & $l_{p} p_{p}, E_{p} t_{p}$ & $1.054571 \times 10^{-34} \frac{\mathrm{kgm}^{2}}{\mathrm{~s}}$ & - \\
\hline Gravitational const & $G$ & - & $\frac{l_{p}^{3}}{m_{p} t_{p}^{2}}$ & $\frac{l_{p}}{m_{p}} c^{2}$ & $6.67430 \times 10^{-11} \frac{\mathrm{m}^{3}}{\mathrm{kgs}^{2}}$ & - \\
\hline Speed of light & $c$ & $\frac{l_{p}}{t_{p}}$ & $\frac{l_{p}}{t_{p}}$ & - & $299,792,458 \frac{\mathrm{m}}{\mathrm{s}}$ & - \\
\hline Planck charge & $q_{p}$ & $\sqrt{4 \pi \epsilon_{0} \hbar c}[4,8,10]$ & $t_{p}$ & - & $5.391247 \times 10^{-44} s$ & $1.876 \times 10^{-18} C$ \\
\hline Elementary charge & $e, q$ & $\frac{4 \pi \hbar \alpha}{\mu_{0} c}[11]$ & $t_{p} \sqrt{\alpha}$ & - & $4.605448 \times 10^{-45} s$ & $1.602 \times 10^{-19} C$ \\
\hline Electric permittivity & $\epsilon_{0}$ & $\frac{1}{\mu_{0} c^{2}}[11]$ & $\frac{t_{p}^{4}}{4 \pi l_{p}^{3} m_{p}}$ & $\frac{t_{p}}{4 \pi p_{p} c^{2}}$ & $7.315968 \times 10^{-63} \frac{\mathrm{s}^{4}}{\mathrm{kgm}^{3}}$ & $8.854 \times 10^{-12} \frac{F}{m}$ \\
\hline Coulomb const & $k_{e}$ & $\frac{1}{4 \pi \epsilon_{0}}$ & $\frac{l_{p}^{3} m_{p}}{t_{p}^{4}}$ & $\frac{p_{p}}{t_{p}} c^{2}$ & $1.087723 \times 10^{61} \frac{\mathrm{kgm}^{3}}{\mathrm{~s}^{4}}$ & $8.988 \times 10^{9} \frac{\mathrm{Nm}^{2}}{\mathrm{C}^{2}}$ \\
\hline \multirow[t]{2}{*}{ Magnetic permeability } & $\mu_{0}$ & $\frac{4 \pi \alpha \hbar}{e^{2} c}[11]$ & $4 \pi \frac{l_{p} m_{p}}{t_{p}^{2}}$ & $4 \pi \frac{p_{p}}{t_{p}}$ & $1.520851 \times 10^{45} \frac{\mathrm{kgm}}{\mathrm{s}^{2}}$ & $1.256 \times 10^{-6} \frac{\mathrm{N}}{A^{2}}$ \\
\hline & $V_{p}$ & $\sqrt{\frac{c^{4}}{4 \pi G \epsilon_{0}}}[12]$ & $\frac{l_{p}^{2} m_{p}}{t_{p}^{3}}$ & $\frac{E_{p}}{t_{p}}, \frac{E_{p}}{q_{p}}$ & $3.628253 \times 10^{52} \frac{\mathrm{kgm}^{2}}{\mathrm{~s}^{3}}$ & $1.043 \times 10^{27} V$ \\
\hline Current & $I_{p}$ & $\sqrt{\frac{4 \pi \epsilon_{0} c^{6}}{G}}[12]$ & $\frac{t_{p}}{t_{p}}$ & $\frac{q_{p}}{t_{p}}, \frac{t_{p}}{q_{p}}$ & 1 & $3.479 \times 10^{25} A$ \\
\hline Inductance & $L_{p}$ & $\frac{1}{4 \pi \epsilon_{0}} \sqrt{\frac{\hbar G}{c^{7}}}[12]$ & $\frac{l_{p}^{2} m_{p}}{t_{p}^{2}}$ & $E_{p}$ & $1,956,081,000 \frac{\mathrm{kgm}^{2}}{\mathrm{~s}^{2}}$ & $1.616 \times 10^{-42} H$ \\
\hline Magnetic inductance & $B_{p}$ & $\sqrt{\frac{c^{5}}{4 \pi \epsilon_{0} \hbar G^{2}}}[12]$ & $\frac{m_{p}}{t_{p}^{2}}$ & $\frac{p_{p}}{l_{p} t_{p}}$ & $7.488021 \times 10^{78} \frac{\mathrm{kg}}{\mathrm{s}^{2}}$ & $2.152 \times 10^{53} T$ \\
\hline
\end{tabular}




\begin{tabular}{|c|c|c|c|c|c|c|}
\hline Constant & Sym & Std form & Elem form & Alt forms & MKS value & EM value \\
\hline \multirow[b]{3}{*}{ Impedance of vacuum } & $G_{p}$ & $4 \pi \epsilon_{0} c$ & $\frac{t_{p}^{3}}{l_{p}^{2} m_{p}}$ & $\frac{t_{p}}{E_{p}}, \frac{q_{p}}{E_{p}}$ & $2.756147 \times 10^{-53} \frac{\mathrm{s}^{3}}{\mathrm{kgm}^{2}}$ & $0.03336 S$ \\
\hline & $Z_{p}$ & $\frac{1}{4 \pi \epsilon_{0} c}[12]$ & $\frac{l_{p}^{2} m_{p}}{t_{p}^{3}}$ & $\frac{E_{p}}{t_{p}}, \frac{E_{p}}{q_{p}}$ & $3.628253 \times 10^{52} \frac{\mathrm{kgm}^{2}}{\mathrm{~s}^{3}}$ & $29.98 \Omega$ \\
\hline & $Z_{0}$ & $\mu_{0} c[11]$ & $4 \pi \frac{l_{p}^{2} m_{p}}{t_{p}^{3}}$ & $4 \pi \frac{E_{p}}{t_{p}}$ & $4.559397 \times 10^{53} \frac{\mathrm{kgm}^{2}}{\mathrm{~s}^{3}}$ & $376.7 \Omega$ \\
\hline Capacitance & $C_{p}$ & $4 \pi \epsilon_{0} \sqrt{\frac{\hbar G}{c^{3}}}[12]$ & $\frac{t_{p}^{4}}{l_{p}^{2} m_{p}}$ & $\frac{t_{p}^{2}}{E_{p}}$ & $1.485907 \times 10^{-96} \frac{\mathrm{s}^{4}}{\mathrm{kgm}^{2}}$ & $1.798326 \times 10^{-45} \mathrm{~F}$ \\
\hline Fine structure & $\alpha$ & $\frac{e^{2}}{4 \pi \epsilon_{0} \hbar c}[11]$ & - & - & .0072973525693 & - \\
\hline Rydberg constant & $R_{\infty}$ & $\frac{\alpha^{2} m_{e} c}{2 h}[11]$ & $\frac{m_{e} \alpha^{2}}{4 \pi l_{p} m_{p}}$ & - & $10973732 \frac{1}{m}$ & - \\
\hline Rydberg energy & $h c R_{\infty}$ & $\frac{1}{2} m_{e} \alpha^{2} c^{2}[11]$ & $\frac{m_{e} \alpha^{2} l_{p}^{2}}{2 t_{p}^{2}}$ & $\frac{1}{2} m_{e} \alpha^{2} c^{2}$ & $2.179872 \times 10^{-18} \frac{\mathrm{kgm}^{2}}{\mathrm{~s}^{2}}$ & - \\
\hline \multirow[t]{2}{*}{ Magnetic flux quantum } & $\phi_{0}$ & $\frac{2 \pi \hbar}{2 e}[11]$ & $\frac{\pi l_{p}^{2} m_{p}}{\sqrt{\alpha} t_{p}^{2}}$ & $\frac{\pi}{\sqrt{\alpha}} E_{p}$ & $7.193730 \times 10^{10} \frac{\mathrm{kgm}^{2}}{\mathrm{~s}^{2}}$ & $2.067834 \times 10^{-15} \mathrm{~Wb}$ \\
\hline & $R_{K}$ & $\frac{2 \pi \hbar}{e^{2}}[11]$ & $\frac{2 \pi m_{p} l_{p}^{2}}{\alpha t_{p}^{3}}$ & $\frac{2 \pi E_{p}}{\alpha t_{p}}$ & $3.124008 \times 10^{55} \frac{\mathrm{kgm}^{2}}{\mathrm{~s}^{3}}$ & $25,812.81 \Omega$ \\
\hline Josephson & $K_{J}$ & $\frac{2 e}{h}[11]$ & $\frac{\sqrt{\alpha} t_{p}^{2}}{\pi l_{p}^{2} m_{p}}$ & $\frac{\sqrt{\alpha}}{\pi E_{p}}$ & $1.390100 \times 10^{-11} \frac{s^{2}}{\mathrm{kgm}^{2}}$ & $4.835978 \times 10^{14} \frac{\mathrm{Hz}}{\mathrm{V}}$ \\
\hline Bohr magneton & $\mu_{B}$ & $\frac{e \hbar}{2 m_{e}}[11]$ & $\frac{\sqrt{\alpha}}{2} \frac{m_{p}}{m_{e}} l_{p}^{2}$ & - & $2.665808 \times 10^{-49} \mathrm{~m}^{2}$ & $9.274010 \times 10^{-24} \frac{J}{T}$ \\
\hline Fermi coupling & & $\frac{G_{F}}{\hbar^{3} c^{3}}[11]$ & $\frac{G_{F} t_{p}^{6}}{l_{p}^{9} m_{p}^{3}}$ & $\frac{G_{F}}{l_{p}^{3} E_{p}^{3}}$ & $1.166379 \times 10^{-5} \mathrm{Ge} / \mathrm{V}^{2}$ & - \\
\hline Conductance quantum & $G_{0}$ & $\frac{2 e^{2}}{2 \pi \hbar}[11]$ & $\frac{\alpha t_{p}^{3}}{\pi l_{p}^{2} m_{p}}$ & $\frac{\alpha t_{p}}{\pi E_{p}}$ & $6.402033 \times 10^{-56} \frac{\mathrm{s}^{3}}{\mathrm{kgm}^{2}}$ & $7.748092 \times 10^{-5} S$ \\
\hline Linear mass density & $\frac{l_{P}}{m_{P}}$ & - & $\frac{l_{p}}{m_{p}}$ & - & $7.426161 \times 10^{-28} \frac{\mathrm{m}}{\mathrm{kg}}$ & - \\
\hline Quantum constant & $l_{P} m_{P}$ & - & $l_{p} m_{p}$ & - & $3.517672 \times 10^{-43} \mathrm{kgm}$ & - \\
\hline Force & $F_{p}$ & $\frac{c^{4}}{G}$ & $\frac{m_{p} l_{p}}{t_{p}^{2}}$ & $\frac{p_{p}}{t_{p}}, \frac{E_{p}}{l_{p}}$ & $1.210255 \times 10^{44} \frac{\mathrm{kgm}^{2}}{\mathrm{~s}^{2}}$ & - \\
\hline Acceleration & $a_{p}$ & $\sqrt{\frac{c^{7}}{\hbar G}}$ & $\frac{l_{p}}{t_{p}^{2}}$ & $\frac{c}{t_{p}}$ & $5.560725 \times 10^{51} \frac{\mathrm{m}}{\mathrm{s}^{2}}$ & - \\
\hline
\end{tabular}

$\alpha=$ Fine structure constant; $\mathrm{A}=$ ampere; $\mathrm{C}=$ coulomb $E_{P}=\mathrm{Planck}$ energy; $\mathrm{F}=$ farad; $\mathrm{H}=$ henry; $\mathrm{kg}=$ kilogram; $l_{P}=$ Planck length; $\mathrm{m}=$ meter; $m_{e}=$ electron mass; $m_{P}=$ Planck mass; $p_{P}=$ Planck momentum; N=newton; s=second; $t_{P}=$ Planck time; $\mathrm{S}=$ siemens; $\mathrm{T}=$ tesla; $\mathrm{W}=$ weber; $\mathrm{V}=$ volt; $\Omega=\mathrm{ohm}$ 


\section{Functions and Equations}

The benefits of replacing traditional constants with Planck units becomes clear in the reformulated versions of physical equations. Each of the following equations substitutes Planck length, mass, and time for $\hbar, G$, and the Coulomb constant $k_{e}$. Insights gained from the equations are then refined into a new physical model that is consistent with experimental observations while offering new insights into quantum mechanics, electromagnetism, and gravity.

\subsection{Photon Energy}

The equation for photon energy

$$
E=h f
$$

can be expressed in Planck units according to equation 1. Substituting $2 \pi l_{P} m_{P} c$ for $h$, and $c / \lambda$ for $f$ gives

$$
E=2 \pi l_{P} m_{P} c\left(\frac{c}{\lambda}\right)
$$

The equation can be regrouped and simplified to emphasize important ratios

$$
\begin{aligned}
E & =\left(\frac{l_{P}}{\lambda}\right) m_{P} c^{2} \\
& =\left(\frac{l_{P}}{\lambda}\right) E_{P}
\end{aligned}
$$

where $\lambda=2 \pi \lambda$. The restated equation reveals how Planck's constant transforms a photon's wavelength into a specific quantity of energy. Out of the fundamental Planck units concealed within Planck's constant, a dimensionless operator is generated from the ratio of Planck length to photon wavelength. The operator reduces the maximum quantity of Planck energy into the correct amount. We can represent the proportionality operator using the symbol $\beta_{\lambda}$ which simplifies the function to

$$
E=\beta_{\lambda} E_{P}
$$

where $\beta_{\lambda}$ is the reduction due to the photon's wavelength and $E_{P}$ is the Planck energy.

\subsection{Compton Wavelength}

The reduced Compton wavelength formula

$$
\lambda_{c}=\frac{\hbar}{m_{0} c}
$$

can be re-stated in Planck units as

$$
\lambda_{c}=\frac{l_{P} m_{P} c}{m_{0} c} .
$$


Grouping related terms again highlights the key proportionalities

$$
\begin{aligned}
\lambda_{c} & =\left(\frac{m_{P}}{m_{0}}\right)\left(\frac{c}{c}\right) l_{P} \\
& =\left(\frac{m_{P}}{m_{0}}\right) l_{P}
\end{aligned}
$$

where $m_{0}$ is the rest mass.

The value of $c$ embedded in Planck's constant isn't required by the Compton wavelength formula, so the function removes it with a factor of $c$ in the denominator. What remains is a dimensionless operator in the proportion of rest mass to Planck mass. This operator acts on the Planck length producing the proper wavelength.

We can represent the simplified operation as

$$
\lambda_{c}=\frac{l_{P}}{\beta_{m}}
$$

where $\beta_{m}$ represents the ratio of rest mass to Planck mass.

\section{3 de Broglie Wavelength}

The de Broglie formula adds a second operator to the mass operator found in the Compton formula. The reduced de Broglie formula

$$
\lambda=\frac{\hbar}{m_{0} v}
$$

can be written in Planck units as

$$
\lambda=\left(\frac{m_{P}}{m_{0}}\right)\left(\frac{c}{v}\right) l_{P}
$$

The de Broglie formula has the same mass operator as the Compton wavelength formula, but instead of removing the value of $c$ in the numerator, it generates a second operator in the ratio of $c$ to $v$. The two operators act on the Planck length producing the wavelength of a massive particle in motion. The de Broglie function can be summarized as

$$
\lambda=\frac{l_{P}}{\beta_{m} \beta_{v}}
$$

where $\beta_{m}$ represents the ratio of rest mass to Planck mass, and $\beta_{v}$ is the ratio of velocity to the speed of light. 


\subsection{Schwarzschild Radius}

The Schwarzschild radius is calculated using the gravitational constant. Equations 1 and 2 show that either $\hbar$ or $G$ could be used with the right modifications. We can convert between the two constants in the following way

$$
G=\frac{l_{P}}{m_{P}} c^{2}=\left(\frac{c}{m_{P}^{2}}\right) l_{P} m_{P} c=\frac{c}{m_{P}^{2}} \hbar .
$$

The gravitational constant behaves like Planck's constant in that it produces operators from the ratios of inputs to Planck units embedded in the constant. The formula for determining the Schwarzschild radius

can be stated in Planck units as

$$
r_{s}=\frac{2 G M}{c^{2}}
$$

$$
\begin{aligned}
r_{s} & =2\left(\frac{M}{m_{P}}\right)\left(\frac{c}{c}\right)\left(\frac{c}{c}\right) l_{P} \\
& =2\left(\frac{M}{m_{P}}\right) l_{P} .
\end{aligned}
$$

The Schwarzschild radius formula doesn't require two values of $c$ embedded in the Gravitational constant, so it removes them with two values of $c$ in the denominator. What remains is a mass operator acting on the Planck length. The restated function demonstrates how the simple proportion of a gravitational body's mass to the Planck mass determines its Schwarzschild radius.

\subsection{Black Hole Temperature}

The equation for black hole temperature

$$
T=\frac{\hbar c^{3}}{8 \pi G k_{B} M}
$$

can be written in terms of energy

$$
k_{B} T=\frac{\hbar c^{3}}{G} \frac{1}{8 \pi M}
$$

and stated in Planck units as

$$
E=\frac{\left(l_{P} m_{P} c\right) c^{3}}{\left(\frac{l_{P} c^{2}}{m_{P}}\right)} \frac{1}{8 \pi M}
$$

The simplified equation

$$
E=m_{P}^{2} c^{2} \frac{1}{8 \pi M}
$$

can be written as a mass operator and a constant acting on the Planck energy

$$
E=\frac{1}{8 \pi}\left(\frac{m_{P}}{M}\right) E_{P}
$$

The restated formula gives a more intuitive explanation of black hole thermodynamics than we get from the traditional constants. 
2.6 Coulomb's Law

Coulomb's law

$$
F=k_{e} \frac{q_{1} q_{2}}{r^{2}}
$$

can be written as a function in natural units

$$
\begin{aligned}
F & =\frac{p_{P}}{t_{P}} c^{2} \frac{t_{1} t_{2}}{l^{2}} \\
& =F_{P} c^{2} \frac{t_{1} t_{2}}{l^{2}}
\end{aligned}
$$

where $F_{P}$ is the Planck Force.

The translation of traditional constants into Planck units in table 2 reveals that electric charge is naturally quantified in units of time. Using the coulomb unit conversion in table 12 , charge is entered in units of seconds and distance in meters. Pairing the inputs with corresponding Planck units found in $c^{2}$ embedded in the Coulomb constant yields four operators acting on the Planck force

$$
F=\left(\frac{l_{P}}{l}\right)\left(\frac{t}{t_{P}}\right)\left(\frac{l_{P}}{l}\right)\left(\frac{t}{t_{P}}\right) F_{P} .
$$

The four operators can be restated using the relationship

$$
\frac{c}{v}=\frac{\frac{l_{P}}{t_{P}}}{\frac{l}{t}}=\left(\frac{l_{P}}{l}\right)\left(\frac{t}{t_{P}}\right)
$$

and simplified as

$$
F=\left(\frac{c^{2}}{v^{2}}\right) F_{P}
$$

Here we see that Coulomb's constant behaves like the other functions; it contains the embedded value $c^{2}$ to formulate operators out of inputs of charge and distance which act on the Planck force. The operator $c^{2} / v^{2}$ and the maximum force potential represent a magnificent correspondence with general particle mechanics. Furthermore, the quantification of electric charge in seconds suggests a mechanical foundation underlying electromagnetism that is hidden by traditional coulomb units.

\section{A New Foundations Model of Physics}

\subsection{Planck Potentials}

The equations in section 2 reveal a general formula for calculating the physical properties of elementary particles and systems. This formula establishes a basis in the maximum potentials of certain physical properties quantified by the Planck units. The function transforms inputs of measured values into one or more proportionality operators based on their ratios to the Planck unit values. The magic of the function is that a simple reduction from the maximum potential determines the output.

The three fundamental Planck units of length, mass, and time represent maximum potentials, and combinations of these units form additional potentials. Each Planck unit or 
combination of units has a finite limit at its maximum potential, and an arbitrarily small potential asymptotically approaching zero. The finite, maximum potential provides a definitive basis for calculating physical properties whereas the asymptotic limit offers no basis. By setting the maximum potential equal to one, any unit distance from the Planck scale can be quantified as the ratio of one divided by the distance. The versatility of this ratio is its applicability across different unit types.

Figure 1 illustrates the maximum potentials of length, mass, and time. In SI units, quantities of length and time are inversely proportional to their potentials while mass is proportional to its potential. Section 2 showcased the simplicity and versatility of the Planck

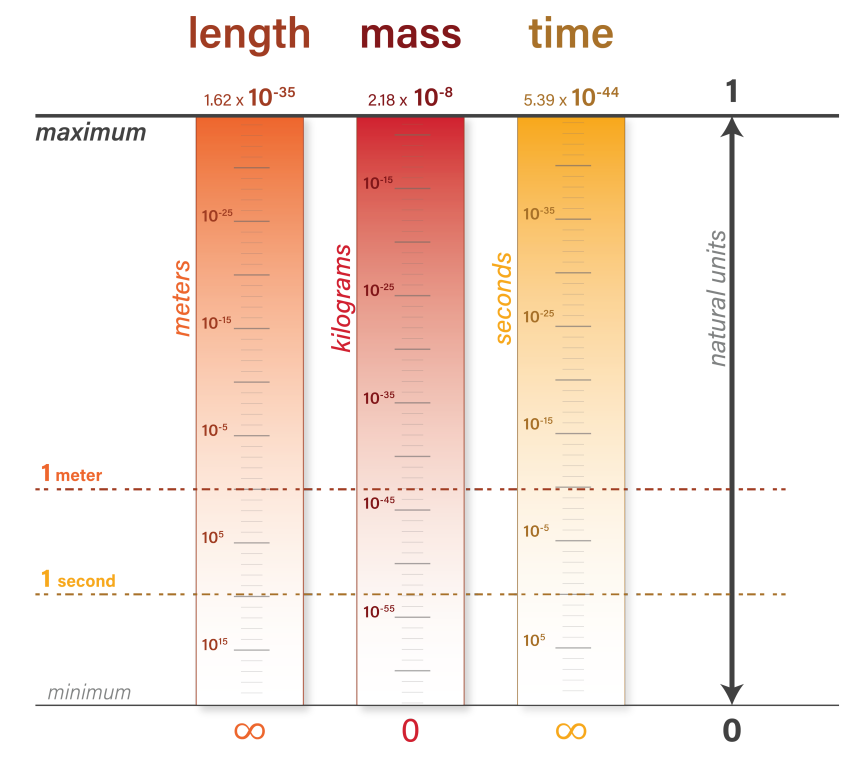

Fig. 1: Planck units represent maximum potentials. Length, mass, and time have definitive maximum potentials at the Planck scale and arbitrarily small potentials asymptotically approaching zero

units in explaining the physical universe. Equations written in terms of these fundamental units yield new insights into physical mechanics previously hidden by the larger structure of composite constants.

The following sections propose a New Foundations Model of physics incorporating the principles of maximum potential and proportionality into the descriptions of quantum mechanics, gravity, and electromagnetism. It attempts to explain physical dynamics using the additional information found in the relationships between length, mass, and time. In addition, the model attempts to reduce the level of abstraction found in descriptions of quantum mechanics that rely predominantly on mathematical formulations.

The model does not try to solve - or even address - the measurement problem. Rather, it presumes that the properties of elementary particles characterized mathematically by wave functions and matrices are genuine physical properties, and not abstract quantities in configuration space. The formulas and explanations describe the fluid, deterministic evolution of particle oscillations without incorporating the stochastic localization brought on by obser- 
vation and measurement. The physical descriptions, therefore, cannot be directly measured, but are inferred from equations and from the statistics of multiple measurements.

The model has shortcomings. Its scope is limited to physical properties in their simplest form-without incorporating complex interactions encountered in all but the most isolated experiments. But just as the Bohr model of the hydrogen atom revealed underlying principles on which more complex descriptions of the atom were later constructed, a New Foundations Model framed in terms of the Planck units may inform more complex theories with fewer gaps subject to interpretation.

I'll introduce the model using the basic mechanics of elementary particles. A universal mechanics function describes the maximum potential of elementary particles, and four proportionality operators reduce this potential into everyday quantities of mass, momentum, and energy. I'll also define two symmetries that conserve maximum potentials and constrain the relationships between quantities of length, mass, and time. Physical dynamics including action and force emerge from these constraints.

Following a demonstration of the mechanical principles, I'll introduce the gravitational and electromagnetic functions. I'll show that gravitational and electromagnetic field potentials share the same maximum potentials as particle mechanics, and the same principles of proportionality determine the strengths of the potentials. I'll finish by comparing the relative strengths of the different forces in terms of their proportionality operators.

The function describing basic mechanical properties of the elementary particles is

$$
\text { Energy }=\mathbf{m}_{\mathbf{P}}\left(\frac{\mathbf{l}_{\mathbf{P}}}{\mathbf{t}_{\mathbf{P}}}\right)\left(\frac{\mathbf{l}_{\mathbf{P}}}{\mathbf{t}_{\mathbf{P}}}\right) \text {. }
$$

The function consists of three parts progressively known as mass, momentum, and energy.

\subsection{Mass}

The relationship between mass and wavelength is described by the Compton and de Broglie formulas in section 2. Compton scattering gives the wavelength of a photon with energy equal to the rest mass of a massive particle [13-15], while the de Broglie formula gives the wavelength of the massive particle. The two formulas coincide at the limit of the massive particle's maximum velocity potential $c$. According to equations 5 and 6 , the massive particle's wavelength equals the photon wavelength when each is traveling at a rate of $c$.

Re-arranging 5 provides the relationship between a particle's rest mass and its Compton wavelength, following from the new definition of Planck's constant

$$
\lambda_{C} m_{0}=l_{P} m_{P}
$$

It is evident that this relationship holds for the rest masses and Compton wavelengths of the charged leptons. Table 3 gives the reduced Compton wavelengths and rest masses of the electron, muon, and tau particles. The product of the two is equal to the constant $l_{P} m_{P}$, or $3.52 \times 10^{-43} \mathrm{kgm}$ in each case.

We also get from equation 9 a relationship between the proportions of wavelength and mass to their maximum potentials, which in section 2 I showed is instrumental for calculating physical properties. The equation shows that the proportion of Planck length to Compton 
Table 3: The Compton wavelengths and rest masses of the charged leptons conserve the value $3.52 \times 10^{-43}$

\begin{tabular}{lccc}
\hline Lepton & $\lambda_{C}(\mathrm{~m})$ & $m_{0}(\mathrm{~kg})$ & $\lambda_{C} m_{0}(\mathrm{kgm})$ \\
\hline $\mathrm{e}$ & $3.8616 \times 10^{-13}$ & $9.1094 \times 10^{-31}$ & $3.5177 \times 10^{-43}$ \\
$\mu$ & $1.8676 \times 10^{-15}$ & $1.8835 \times 10^{-28}$ & $3.5177 \times 10^{-43}$ \\
$\tau$ & $1.1105 \times 10^{-16}$ & $3.1675 \times 10^{-27}$ & $3.5177 \times 10^{-43}$ \\
\hline
\end{tabular}

wavelength is equal to the proportion of rest mass to Planck mass. Re-arranging 9 gives an equality between these ratios

$$
\frac{l_{P}}{\lambda_{C}}=\frac{m_{0}}{m_{P}} .
$$

According to the de Broglie formula, this equivalence holds true for all combinations of a massive particle's wavelength and momentum. From 6 we get the following relationship for a massive particle

$$
\lambda m_{0}\left(\frac{v}{c}\right)=l_{P} m_{P}
$$

where $t$ is the de Broglie wavelength. Multiplying the rest mass by $v / c$ gives a general formula for the wavelength and mass of a particle at any velocity

$$
\lambda m=l_{P} m_{P}
$$

where $m$ is the rest mass multiplied by $v / c$. This quantity of inertial mass is traditionally treated as momentum in units of $\mathrm{kgm} / \mathrm{s}$, but I'll show the significance of treating it as a form of mass throughout this paper. The inertial mass $m$ is considered a physical property of bosons and fermions quantified by the inverse particle wavelength, and determines the energy of motion in both types of particles. Inertial mass is given here in non-relativistic terms.

It is important to distinguish between the Compton wavelength as a measure of rest energy, quantified by electromagnetic radiation, and the Compton wavelength of a massive particle. As a measure of rest mass, the Compton formula is not giving a physical description of a massive particle's wavelength. That's given in 6 , which shows that the wavelength of a massive particle approaches the Compton wavelength as its velocity approaches $c$.

Given that $l_{P} m_{P}$ is constant, we can quantify the product of wavelength and mass as

$$
\lambda m=3.52 \times 10^{-43} \mathrm{kgm}
$$

where $m$ is the inertial mass.

Since the product of wavelength and mass is invariant to changes in the two terms, the quantity $3.52 \times 10^{-43} \mathrm{kgm}$ is conserved. A fixed quantity of mass-wavelength can be illustrated as an inverse function of the two, shown in figure 2 . We can determine the mass and wavelength of a particle using the formulas

$$
m=\frac{3.52 \times 10^{-43}}{\lambda} \mathrm{kgm}
$$

and

$$
\lambda=\frac{3.52 \times 10^{-43}}{m} \mathrm{kgm} .
$$




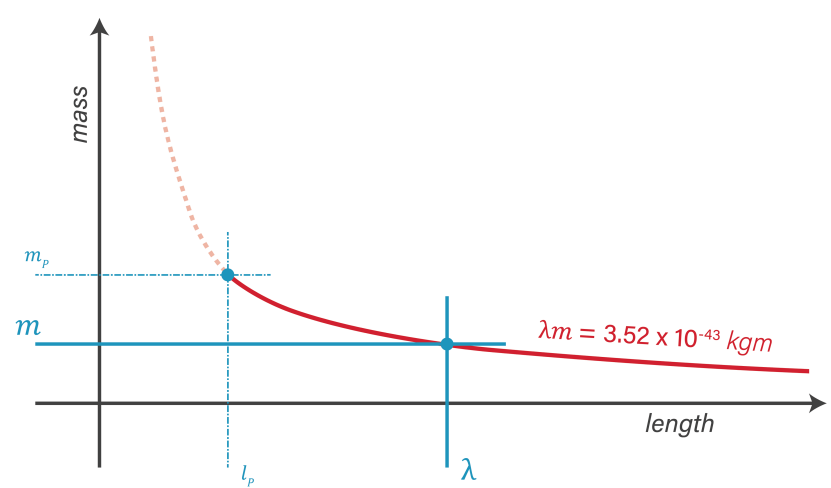

Fig. 2: Particle wavelength and inertial mass are inversely related, conserving the product of wavelength and mass, $3.52 \times 10^{-43} \mathrm{kgm}$.

The curve in figure 2 represents possible combinations of wavelength and mass up to the Planck scale, which is shown at the natural unit coordinate $(1,1)$. From the Planck scale, as units of length increase, units of mass decrease reciprocally so that $m=1 / \lambda$.

The conservation of a particle's mass-wavelength gives a valuable physical constant I call the quantum constant, equal to $3.52 \times 10^{-43} \mathrm{kgm}$. According to this proposal, the relationship between a particle's wavelength and its inertial mass is a conserved property of elementary particles including bosons and fermions.

\subsection{Momentum}

The second component of the particle mechanics function is the maximum ratio of length to time. Combining this ratio with the Planck mass gives the maximum momentum potential

$$
p_{P}=m_{P} \frac{l_{P}}{t_{P}}
$$

Momentum quantifies the inertial strength of both massive and massless particles. The traditional formula for photon momentum

$$
p=\frac{h}{\lambda}
$$

can be re-stated in Planck units as

$$
p=\left(\frac{l_{P}}{\lambda}\right) p_{P}
$$

This form of the equation identifies the Planck momentum embedded in Planck's constant as the maximum momentum potential, which is obtained when the proportionality operator $l_{P} / \lambda$ is equal to one. The operator demands that a photon's momentum is inversely proportional to its wavelength.

At the Planck scale, the maximum momentum potential distributes the conserved quantum constant over the smallest possible distance, determined here using the Planck time. 
From the definition of Planck momentum we can arrange an equality that is useful for describing elementary particle mechanics

$$
l_{P} m_{P}=p_{P} t_{P}
$$

Figure 3 illustrates the relationships between the Planck units in equation 14 including important ratios between them. The figure shows that the relationship between Planck mass

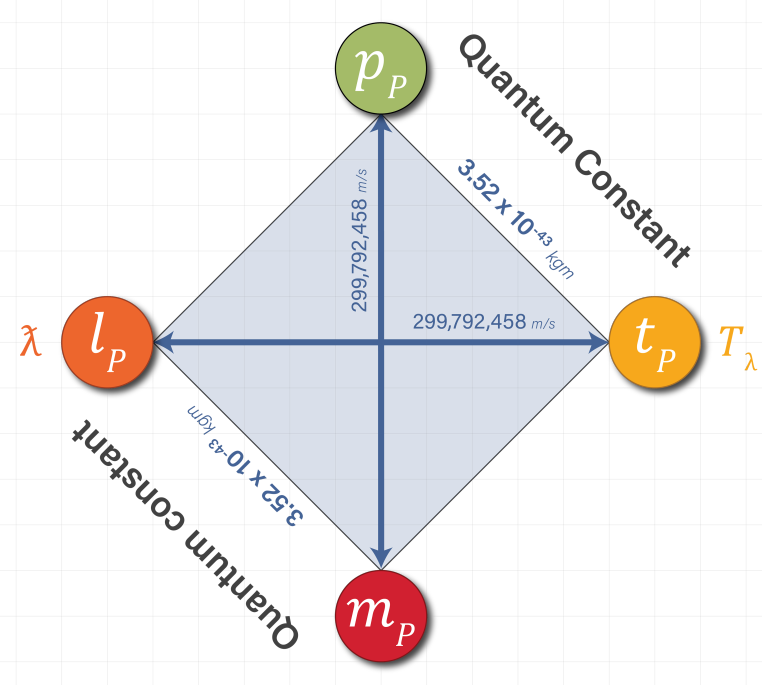

Fig. 3: The relationship between Planck mass and Planck momentum is governed by the speed of light. The product of Planck length and mass is equal to the product of Planck momentum and Planck time.

and Planck momentum is governed by the ratio of Planck length to Planck time- the speed of light. The figure also shows an equivalent relationship between the product of Planck momentum and Planck time, and the product of Planck length and Planck mass. Each of these values is equal to the quantum constant, $3.52 \times 10^{-43} \mathrm{kgm} / \mathrm{s}$.

Equation 14 can be modified to show the relationship between wavelength, mass, time, and momentum at any scale. Replacing $l_{P} m_{P}$ with $\lambda m$, according to 10 , retains the conserved value of the quantum constant in the numerator, so that the particle's momentum is determined in units of $\mathrm{kgm} / \mathrm{s}$ by the time component in the denominator. A consistent definition of momentum for particles with and without rest mass follows when we treat momentum as quantifying a particle's spatial distribution without regard to its velocity. In the next section I'll show why momentum was made to align with velocity in dimensions of $\mathrm{kgm} / \mathrm{s}$.

To establish a consistent description of momentum for massive and massless particles, I'll refer to the time component of momentum as $T_{\lambda}$, defined as $\lambda / c$. For particles with no rest mass, $T_{\lambda}$ is equal to the particle's period $T$.

According to the proposed model, the following relationship is applicable at all scales and represents a fundamental symmetry of nature in the conservation of mass and momentum

$$
\lambda m=p T_{\lambda}
$$


where $\lambda$ is particle wavelength, $m$ is the corresponding mass, and $T_{\lambda}$ re-quantifies the inertial mass in traditional units of momentum. Because $p$ is the relationship between the other three quantities, and because the quantum constant $\lambda m$ is conserved, the quantity $p T_{\lambda}$ is also conserved.

We can arrange 15 into a definition of momentum using the three physical quantities of length, mass, and time

$$
p=\frac{\lambda m}{T_{\lambda}} \mathrm{kgm} / \mathrm{s}
$$

\section{Wavelength Operator}

In sections 2 and 3.3, I showed that the operator $l_{P} / \lambda$ reduces the Planck momentum and Planck energy in the right proportions to produce a photon's momentum and energy. This operator is defined as $\beta_{\lambda}$, the wavelength operator. Figure 4 illustrates how the wavelength operator transforms a photon proportionally. The operator modifies each of the terms by the

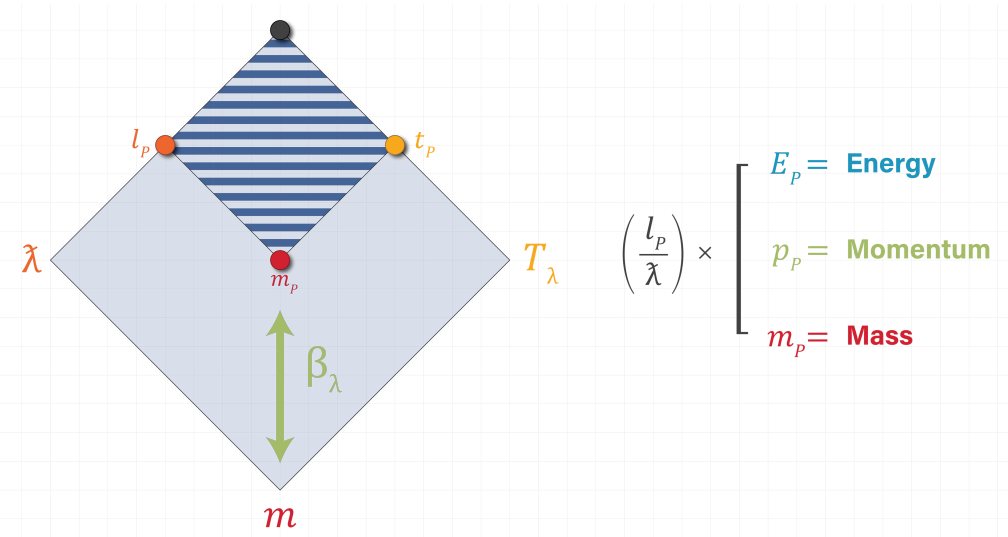

Fig. 4: Electromagnetic radiation is quantized by a reduction operator applied to the maximum momentum and energy potentials. The operator modifies a photon's wavelength mass, and time, producing proportional quantities of momentum and energy.

same ratio, increasing the photon's wavelength, decreasing its inertial mass (sometimes referred to as effective mass), and increasing $T_{\lambda}$. Because $t m$ is conserved, only one instance of the operator is needed to obtain the right quantity of momentum. However, the conservation of $\lambda m$ shown in 11 tells us that all three values change. We also know that $T_{\lambda}$ must increase with the wavelength to not exceed the speed of light.

While the operator modifies a photon's wavelength, inertial mass, time, momentum, and energy, it does not change its velocity, which remains at the maximum velocity potential $c$.

\subsubsection{Photon Mechanics}

The New Foundations Model can be demonstrated using known properties of photons. Table 4 gives the properties of several known photons, including photons of the Compton wavelength for the electron, muon, and tau particles; a photon emitted during the hyperfine transition of Caesium 133 according to the SI definition of a second; and the average transition of an electron from the first to second orbital of a hydrogen atom. The properties of 
each photon were calculated by applying the wavelength operator $\beta_{\lambda}\left(l_{P} / \lambda\right)$ to Planck units of length, mass, time, momentum, and energy.

Table 4: Photon properties are calculated by applying the wavelength operator $l_{P} / \lambda$ to Planck units of length, mass, time, momentum, and energy. Values agree with standard formulas. The quantum constant $\lambda m$ is conserved in each case.

\begin{tabular}{lccccccc}
\hline Photon & $\beta_{\lambda}\left(l_{P} / \lambda\right)$ & $\lambda(\mathrm{m})$ & $m(\mathrm{~kg})$ & $T_{\lambda}, T(\mathrm{~s})$ & $p(\mathrm{kgm} / \mathrm{s})$ & $E\left(\mathrm{kgm}^{2} / \mathrm{s}^{2}\right)$ & $\lambda m, p T_{\lambda}$ \\
\hline$\lambda_{P}$ & 1 & $1.62 \times 10^{-35}$ & $2.18 \times 10^{-8}$ & $5.39 \times 10^{-44}$ & 6.52 & $1.96 \times 10^{9}$ & $l_{P} m_{P}$ \\
$\lambda_{C}, \tau$ & $1.46 \times 10^{-19}$ & $1.11 \times 10^{-16}$ & $3.17 \times 10^{-27}$ & $3.70 \times 10^{-25}$ & $9.50 \times 10^{-19}$ & $2.85 \times 10^{-10}$ & $l_{P} m_{P}$ \\
$\lambda_{C}, \mu$ & $8.65 \times 10^{-21}$ & $1.87 \times 10^{-15}$ & $1.88 \times 10^{-28}$ & $6.23 \times 10^{-24}$ & $5.65 \times 10^{-20}$ & $1.69 \times 10^{-11}$ & $l_{P} m_{P}$ \\
$\lambda_{C}$ & $4.19 \times 10^{-23}$ & $3.86 \times 10^{-13}$ & $9.11 \times 10^{-31}$ & $1.29 \times 10^{-21}$ & $2.73 \times 10^{-22}$ & $8.19 \times 10^{-14}$ & $l_{P} m_{P}$ \\
$\lambda_{1 s-2 p}$ & $1.33 \times 10^{-28}$ & $1.22 \times 10^{-7}$ & $2.89 \times 10^{-36}$ & $4.06 \times 10^{-16}$ & $8.67 \times 10^{-28}$ & $2.60 \times 10^{-19}$ & $l_{P} m_{P}$ \\
$\Delta v_{C s}$ & $4.96 \times 10^{-34}$ & $3.26 \times 10^{-2}$ & $1.08 \times 10^{-41}$ & $1.09 \times 10^{-10}$ & $3.23 \times 10^{-33}$ & $9.69 \times 10^{-25}$ & $l_{P} m_{P}$ \\
\hline
\end{tabular}

Applying the wavelength operator $\beta_{\lambda}$ to the Planck units produces values of momentum and energy that agree with traditional formulas. The table shows that values of photon momentum and energy are accounted for entirely by the wavelength operator, while the photon's velocity remains constant at the maximum velocity potential $c$. The table also shows that $\lambda m$ and $p T_{\lambda}$ are conserved for each photon according to 15 .

The presence of a mass term in the equations is interesting. While photons do not interact with the Higgs field in the way that fermions and $W \pm$ and $Z_{0}$ bosons acquire rest mass, the term offers evidence for a broader definition of mass inclusive of inertial mass. There are compelling reasons to do so $[13,16]$, including

1. Einstein's formula for mass energy equivalence, $E=m c^{2}$ produces the correct energy of a photon when we use the inertial mass in table 4.

2. The conservation of mass-wavelength in 10 and 15 is valid for bosons and fermions with respect to inertial mass. We can treat inertial mass as an underlying symmetry that is partially broken with rest mass, which bifurcates a particle's kinetic energy of motion and its latent rest energy.

3. Planck's constant, which is used in equations determining the momentum and energy of massive and massless particles, includes a quantity of Planck mass as shown in equation 1 .

4. According to the New Foundations Model, the proportionality operator $l_{P} / \lambda$ affects quantities of length, mass, and time in the same proportions. The collective consistency of this approach is demonstrated throughout the model.

Figure 4 illustrates in a conceptual way the attributes of a photon according to 15 . The wavelength operator $l_{P} / \lambda$ quantifies an increase in wavelength from the minimum Planck length. As the illustration shows, the resulting wavelength corresponds with reduced quantities of mass, momentum, time, and energy from their maximum potentials. Meanwhile, the photon's velocity remains at a constant rate of $c$.

\subsection{Energy}

The third component of the particle mechanics function is another instance of Planck length to Planck time. Multiplying this final component to the Planck momentum yields the maxi- 


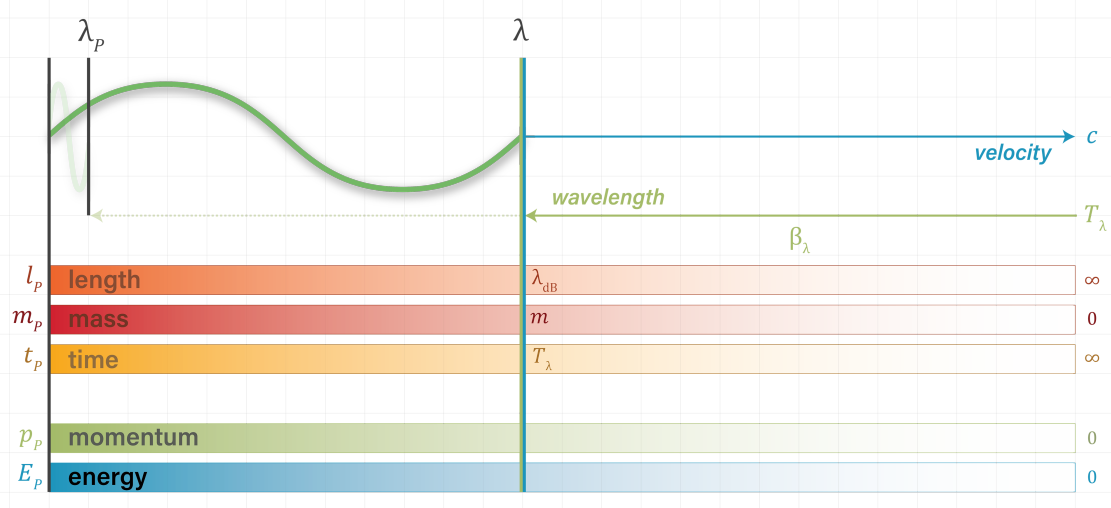

Fig. 5: Photon properties are determined using the wavelength proportionality operator. An increase in wavelength is proportional to decreases in mass, momentum, and energy

mum energy potential

$$
E_{P}=p_{P} \frac{l_{P}}{t_{P}}
$$

This second factor of $l_{P} / t_{P}$ adds the dynamic property of motion. In Planck units, it represents the maximum rate of displacement in a particle's position with respect to time. Energy combines the change in wavelength, described by momentum, with its rate of displacement. The resulting energy is a dynamic redistribution of the particle over space and time.

From the definition of Planck energy we get an equality between Planck momentum and Planck energy in the form

$$
l_{P} p_{P}=E_{P} t_{P}
$$

Figure 6 illustrates the relationship between momentum and energy in equation 16. As the figure shows, the Planck ratios of energy to momentum and length to time are equal to $c$. The figure also shows the equivalent relationships between the product of Planck energy and time, and the product of Planck momentum and length. Each of these quantities is equal to Planck's constant, $1.05 \times 10^{-34} \mathrm{kgm}^{2} / \mathrm{s}$.

Equation 16 expresses two important physical concepts. Each side of 16 represents a form of action in dimensions of momentum-length and energy-time, both in units of $\mathrm{kgm}^{2} / \mathrm{s}$. To get the second concept, we can re-arrange 16 as

$$
\frac{p_{P}}{t_{P}}=\frac{E_{P}}{l_{P}}
$$

to produce two equivalent forms of force in dimensions of momentum per time and energy per length, both in units of $\mathrm{kgm} / \mathrm{s}^{2}$.

Equation 16 can be modified to quantify momentum and energy at different scales. Applying proportionality operators to the maximum Planck energy potential produces

$$
\Delta x p=\Delta t E_{k}
$$

where $\Delta x$ represents the rate of change in an oscillating particle's position and $\Delta t$ represents the corresponding time. Selecting the particle's wavelength $\lambda$ as the change in position gives 


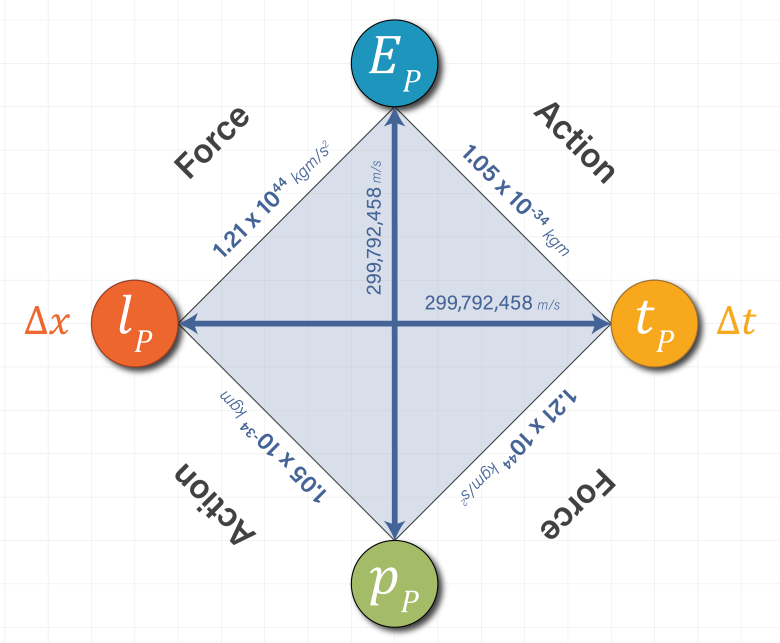

Fig. 6: The momentum-energy relationship repeats the mass-momentum relationship in the ratio of Planck length to time. The ratios between Planck units in the illustration define the physical dynamics of action and force.

the oscillation period $T$ as the change in time. Restating the equation in terms of oscillation period gives

$$
\lambda p=T E_{k} .
$$

Just as $\lambda m$ and $p T_{\lambda}$ are conserved quantities in the quantum constant, $\lambda p$ and $T E_{k}$ are conserved quantities in the amount of Planck's constant. Equality 19 is proposed as a second, fundamental symmetry of nature that conserves momentum and energy.

\subsubsection{Rest Mass}

Determining the properties of photons is simple in that a single proportionality operator transforms the maximum momentum and energy potentials into measured values of a given photon. Particles that have rest mass are more complex due to their interaction with the Higgs field. This interaction produces a quantity of latent rest mass in addition to the inertial mass linked with the particle's wavelength.

To account for the Higgs interaction, three additional operators are needed.

\section{Rest Mass Operator}

The interaction between a massive particle and the Higgs field reduces the maximum mechanical energy potential from the Planck scale down to the scale of the particle's Compton wavelength. As a result, we can treat the Compton wavelength as analogous to the Planck length - the particle's maximum wavelength potential, or shortest wavelength. The rest mass operator also reduces the maximum potential for generating inertial mass. The quantity of mass associated with the particle's Compton wavelength is equal to the particle's rest mass because the Compton and de Broglie formulas converge at the speed of light.

For particles that acquire rest mass, the model adds a rest mass operator, $\beta_{m}$, in the ratio of the Planck length to the particle's Compton wavelength; or equivalently, in the ratio of its 
rest mass to the Planck mass. The rest mass operator only breaks the symmetry proposed in 10 with respect to the particle's total mass and energy, and does not affect its inertial mass and kinetic energy.

Table 5 shows two equivalent forms of the rest mass operator, $l_{P} / \lambda_{C}$ and $m_{0} / m_{P}$, for each of the charged leptons.

Table 5: Two equivalent forms of the rest mass operator produce expected intervals in the charged leptons' Compton wavelengths and rest masses, demonstrating the equivalence in proportions of length and mass to their maximum potentials.

\begin{tabular}{lccccc}
\hline Lepton & $l_{P} / \lambda, m_{0} / m_{P}$ & $\lambda_{C}(m)$ & Length ratio & $m_{0}(\mathrm{~kg})$ & Mass ratio \\
\hline $\mathrm{e}$ & $4.1855 \times 10^{-23}$ & $3.8616 \times 10^{-13}$ & - & $9.1094 \times 10^{-31}$ & - \\
$\mu$ & $8.6542 \times 10^{-21}$ & $1.8676 \times 10^{-15}$ & 206.77 & $1.8835 \times 10^{-28}$ & 206.77 \\
$\tau$ & $1.4554 \times 10^{-19}$ & $1.1105 \times 10^{-16}$ & 16.817 & $3.1675 \times 10^{-27}$ & 16.817 \\
\hline
\end{tabular}

The rest mass operator $\beta_{m}$ applied to the Planck length and Planck mass yields known values of the leptons' Compton wavelengths and rest masses. In addition, the table shows that intervals of wavelength and mass between generations of leptons are preserved when the operator is applied. For each interval between particles, the change in wavelength matches a change in rest mass.

\section{Velocity Operator}

The Higgs field interaction reduces the rate of change in a particle's position with respect to time. For charged leptons, the model adds a velocity operator, $\beta_{v}$, representing the rate of displacement to time. The rest mass operator changes the scale on which the velocity operator acts-instead of reaching a maximum velocity at the Planck scale, as photons do, the maximum velocity is reached at the Compton scale.

The wavelength operator we applied to photons also applies to particles with rest mass; however, the maximum wavelength potential is also changed from the Planck length to the Compton wavelength. The wavelength and velocity operators are equivalent and become normalized in dimensions of distance per time so that a measurement of a particle's velocity also tells us the change in its wavelength. These two operators create a combined reduction $\beta^{2}$, or $v^{2} / c^{2}$.

The equivalence of these two operators confuses the meaning of momentum when applied to massive particles. To be consistent, the formula $p=m v$ must represent a particle's strength due to its wavelength and not due to its velocity. To treat a massive particle's momentum as a function of velocity is incompatible with massless particle momentum where velocity is always a constant rate of $c$. In the case of photons, a change in momentum is a change in wavelength that increases or decreases the particle's strength proportionally. This definition of momentum can be applied consistently to both bosons and fermions according to 15 . For massive and massless particles, momentum is analogous to a payload quantified by the concentration of wavelength, whereas velocity delivers the payload.

The use of momentum pre-dates the emergence of quantum theory when wave-like attributes of matter first became known. For Newton and others who described momentum prior to the 20th century, the quantity $v^{2}$ simply matched the observational data. Louis de Broglie's introduction of the quantum mechanical formula for momentum clarifies the separate roles of wavelength and velocity in determining quantities of momentum and energy. 
Taken together, the wavelength and velocity operators constitute a 2-part mechanism governing the kinetics of elementary particles. The two operators act together, modifying a particle's wavelength at the same time changing its velocity. Because the operators are equal in magnitude, they produce a squared quantity of kinetic energy relative to either the particle's wavelength or its velocity. Treating these operators as reductions from a maximum potential, we can characterize longer wavelengths and slower velocities as larger dilutions of a conserved quantum constant over space and time. This 2-part mechanism gives a beautifully simple explanation of kinetic energy for massless and massive particles, universally determined by the strengths of wavelength and velocity.

It is now evident how the conversion of inertial mass into units of $\mathrm{kgm} / \mathrm{s}$ requires the introduction of $T_{\lambda}$ into the function. The combined change in wavelength and velocity means that we can calculate the particle's temporal period as

$$
T=T_{\lambda} \frac{\lambda}{\lambda_{C}}=T_{\lambda} \frac{c}{v}=\frac{\lambda}{v} .
$$

\section{Spin Operator}

The three operators for rest mass, wavelength, and velocity explain the basic mechanical properties of massive particles except for a one-half reduction in kinetic energy. The New Foundations Model offers a physical explanation for this reduction as a particle's intrinsic spin, where two half-spin rotations complete an oscillation cycle. According to the proposed model, a $1 / 2$ reduction in kinetic energy is explained as the dilution of energy across an extended cycle. The model presumes that energy is diluted by intrinsic spin and must still be applied to composite particles with aggregated spin attributes.

The addition of a spin operator creates a variation of equation 19 for half-spin particles, giving

$$
\lambda p=2 T E_{k} .
$$

Figure 7 illustrates the relationship between mass, momentum, and energy in terms of the proposed proportionality operators. For particles with rest mass, the maximum potential previously defined as the Planck scale is replaced by the Compton scale. The red diamond represents the reduced maximum potentials determined by applying the rest mass operator to units of Planck length, mass, and time. Reductions in the equivalent wavelength and velocity operators further reduce the particle's momentum and energy, shown in the upper and lower diamonds as dilutions from the maximum momentum and energy potentials. These two operators increase the particle's wavelength and reduce its velocity equally.

The diagram doesn't show the fourth reduction which reduces the kinetic energy in half due to an extended cycle over two half-spin rotations.

\subsection{Putting it All Together}

Equation 20 can now be stated in the following form representing physical quantities of length, mass, and time

$$
K E=\frac{1}{2} m \frac{\lambda}{T_{\lambda}} \frac{\lambda}{T}
$$

The following sections demonstrate the New Foundations Model using known particle properties. While the model reproduces the same quantities as traditional formulas, it explains the mechanics in more physically meaningful terms. In addition, the demonstrations showcase the consistency with which the symmetries, maximum potentials, and operators describe physical laws. 


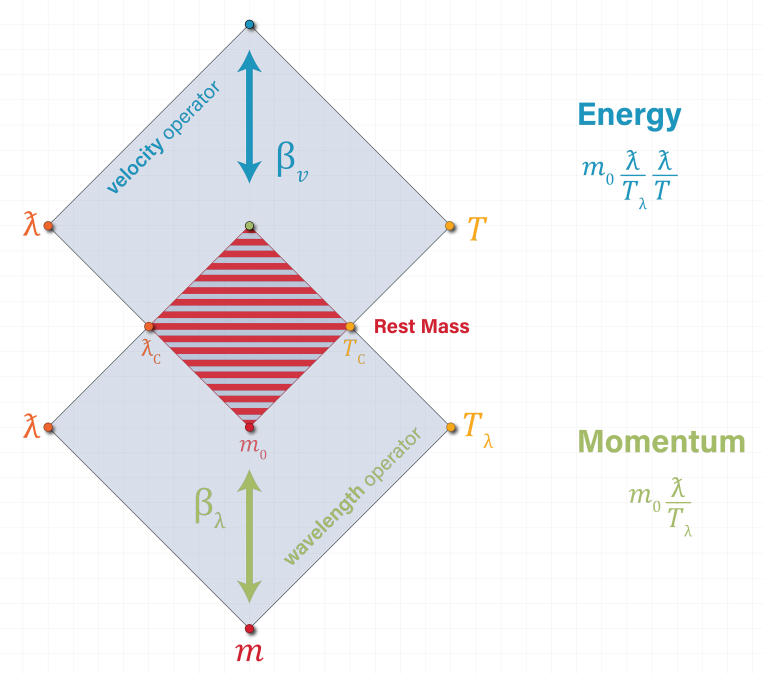

Fig. 7: Charged leptons are reduced from their maximum mass, momentum, and energy potentials by four operators: the rest mass operator, wavelength operator, velocity operator, and spin operator (not shown in the diagram).

\subsubsection{Summary of Symmetries}

Table 6 summarizes the two proposed symmetries. Two equalities express the conservation of mass, momentum, and energy, and define the mechanical properties of action and force.

Table 6: A summary of symmetries accounting for the conservation of mass, momentum, and energy.

\begin{tabular}{ccccc}
\hline Symmetry & Current & Max potential & Invariant & Conserved quantity \\
\hline$\lambda m=p T_{\lambda}$ & & $l_{P} m_{P}$ & $\lambda m$ & $3.52 \times 10^{-43} \mathrm{kgm}$ \\
& & $p_{P} t_{P}$ & $p T_{\lambda}$ & $3.52 \times 10^{-43} \mathrm{kgm}$ \\
& $\lambda / T_{\lambda}$ & $c$ & & \\
\hline$\lambda p=T E_{k}$ & & $l_{P} p_{P}$ & $\lambda p$ & $1.05 \times 10^{-34} \mathrm{kgm}^{2} / \mathrm{s}$ \\
& & $t_{P} E_{P}$ & $T E_{k}$ & $1.05 \times 10^{-34} \mathrm{kgm}^{2} / \mathrm{s}$ \\
& $\Delta x / \Delta t$ & $c$ & & \\
\hline
\end{tabular}

The first symmetry is equation 15 , the relationship between mass and momentum. According to the equality, the product of wavelength and mass is equal to the product of momentum and time. Each side of the equality conserves the quantity $3.52 \times 10^{-43} \mathrm{kgm}$. This symmetry remains unbroken with respect to the kinetic energy of massless and massive particles but is broken by the generation of rest mass separating the kinetic and latent energies of massive particles.

The quantity $3.52 \times 10^{-43} \mathrm{kgm}$ is the proposed quantum constant which is invariant for the product of mass and wavelength. Reductions from the maximum Planck potentials increase 
the spatial and temporal distribution of the quantum constant, diluting its strength in physical interactions.

The second symmetry is equation 19, the relationship between momentum and energy. According to the equality, the product of a change in position and momentum is equal to the product of a change in time and kinetic energy. Each side of the equality conserves the quantity $1.05 \times 10^{-34} \mathrm{kgm}^{2} / \mathrm{s}$, Planck's constant. This symmetry is modified for fermions which undergo a reduction in spin resulting in equation 20 .

\subsubsection{Summary of Operators}

Table 7 summarizes the operators of the particle mechanics function.

Table 7: A summary of the mechanical operators: the rest mass, wavelength, velocity, and spin operators.

\begin{tabular}{lccc}
\hline Operator Name & Symbol & Photon & Charged Leptons \\
\hline Rest Mass Operator & $\beta_{m}$ & - & $\frac{l_{P}}{\lambda_{C}}, \frac{m_{0}}{m_{P}}$ \\
Wavelength Operator & $\beta_{\lambda}$ & $\frac{l_{P}}{\lambda}$ & $\frac{\lambda_{C}}{\lambda}, \frac{m}{m_{0}}, \alpha$ \\
Velocity Operator & $\beta_{v}$ & 1 & $\frac{\lambda t_{P}}{T l_{P}}, \frac{v}{c}$ \\
Spin Operator & $s$ & 1 & $\frac{1}{2}$ \\
\hline
\end{tabular}

$\alpha=$ fine structure constant; $l_{P}=$ Planck length; $\lambda_{C}=$ reduced Compton wavelength; $\lambda=$ particle wavelength; $m_{P}=$ Planck mass; $m_{0}=$ rest mass

According to the New Foundations Model, the four operators in table 7 quantify mechanical properties of elementary particles as reductions from maximum Planck potentials. Only the wavelength operator affects photons, reducing their quantities of mass, momentum, and energy in proportion to the maximum Planck potentials. Photon energy is entirely kinetic and carries no latent energy.

Particles with rest mass add the rest mass, spin, and velocity operators to account for the Higgs interactions. The rest mass operator separates a particle's inertial and latent mass, where inertial mass determines kinetic energy and both forms of mass contribute to the particle's total mechanical energy. The velocity operator is equal to the wavelength operator, and the combination of these two operators creates a squared quantity of kinetic energy. The spin operator reduces the particle's kinetic energy in half.

It is important to note that I've defined the wavelength operator differently for particles that have rest mass from those that do not. For particles with rest mass, wavelength as a physical attribute is represented by the Planck length divided by the product of rest mass and wavelength operators. For particles with no rest mass, the physical attribute is calculated simply as the Planck length divided by the wavelength operator. This convention is used to incorporate rest mass and to highlight the relationship between particle wavelength and velocity. 


\subsubsection{Heisenberg Uncertainty Principle}

The New Foundations Model treats the symmetries in table 6 as ontic descriptions of physical laws. In describing the fluid dynamics of elementary particles, unimpeded by localizing interactions, the symmetries are given as equalities.

Introducing an act of localization invokes the measurement problem, and the equalities in table 6 become inequalities defining the transitional boundaries between a distributed particle state and a localized measurement of some physical property.

Two common forms of the uncertainty principle

$$
\Delta x \Delta p \geq \frac{\hbar}{2}
$$

and

$$
\Delta t \Delta E \geq \frac{\hbar}{2}
$$

can be restated as

$$
\Delta x \Delta p \geq \frac{l_{P} P_{P}}{2}
$$

and

$$
\Delta t \Delta E \geq \frac{t_{P} E_{P}}{2}
$$

The equations can also be written using the conserved, reduced values of Planck's constant $\lambda p$, and $T E$, according to 18 and 19.

The limitations imposed by the inequalities can also be written in terms of proportionalities. Assuming positive rates of change, the inequalities can be arranged as

$$
\frac{\Delta p}{p_{P}} \geq \frac{l_{P}}{\Delta x}
$$

and

$$
\frac{\Delta E}{E_{P}} \geq \frac{t_{P}}{\Delta t}
$$

\subsubsection{Demonstrating the Model with Electrons}

The New Foundations Model can be demonstrated using electron properties. Table 8 applies the four operators to an electron in stages.

The table begins with the maximum Planck potentials included in the particle mechanics function. The top row includes values of Planck length, mass, time, momentum, and energy. The unbroken symmetries from table 6 are also shown.

The second section applies the rest mass operator $\beta_{m}$ which reduces the electron's maximum kinetic potentials from the Planck scale down to the Compton scale.

The third section further reduces the kinetic energy potential by applying the spin operator, reducing the electron's kinetic energy in half.

The final section applies the wavelength and velocity operators. Each row represents a different value of the two equivalent operators on a scale of 0 to 1 , where 1 is the maximum potential in wavelength (the shortest wavelength) and velocity (the greatest velocity). Demonstrated values of the operator include $\alpha$, the fine structure constant, which produces the ground state properties of an electron in the hydrogen atom. 
Table 8: The proposed operators are applied to an electron in steps. From the maximum Planck potentials, reductions are applied for the rest mass operator, the spin operator, and the wavelength and velocity operators.

\begin{tabular}{|c|c|c|c|c|c|c|c|c|}
\hline Max & $l_{P}(m)$ & $m_{P}(k g)$ & $t_{P}(s)$ & $p_{P}(\mathrm{kgm} / \mathrm{s})$ & $E_{P}\left(k m^{2} / s^{2}\right)$ & $\lambda m, p T_{\lambda}$ & $\Delta x p, \Delta t E_{k}$ & $\lambda / T_{\lambda} \Delta x / \Delta t$ \\
\hline 1 & $1.62 \times 10^{-35}$ & $2.18 \times 10^{-8}$ & $5.39 \times 10^{-44}$ & 6.52 & $1.96 \times 10^{9}$ & $l_{P} m_{P}$ & $\hbar$ & $c$ \\
\hline
\end{tabular}

\begin{tabular}{|c|c|c|c|c|c|c|}
\hline$\beta_{m}$ & $\lambda_{C}$ & $m_{0}$ & $T_{\lambda}$ & $p$ & $K E$ & $\lambda m, p T_{\lambda} \Delta x p, \Delta t E_{k} \quad \lambda / T_{\lambda} \Delta x / \Delta t$ \\
\hline $4.19 \times 10^{-23}$ & $3.86 \times 10^{-13}$ & $9.11 \times 10^{-31}$ & $1.29 \times 10^{-21}$ & $2.73 \times 10^{-22}$ & $8.19 \times 10^{-14}$ & $l_{P} m_{P}$ \\
\hline
\end{tabular}

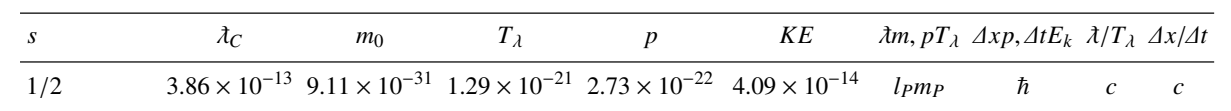

\begin{tabular}{|c|c|c|c|c|c|c|c|c|c|}
\hline$\beta_{\lambda}, \beta_{v}$ & $\lambda$ & $m$ & $T_{\lambda}$ & $p$ & $K E$ & $\lambda m, p T_{\lambda}$ & $\Delta x p, \Delta t E_{k}$ & $\lambda / T_{\lambda}$ & $\Delta x / \Delta t$ \\
\hline 1.0 & $3.86 \times 10^{-13}$ & $9.11 \times 10^{-31}$ & $1.29 \times 10^{-21}$ & $2.73 \times 10^{-22}$ & $4.09 \times 10^{-14}$ & $l_{P} m_{P}$ & $\hbar$ & $c$ & $c$ \\
\hline 0.9 & $4.29 \times 10^{-13}$ & $8.20 \times 10^{-31}$ & $1.43 \times 10^{-21}$ & $2.46 \times 10^{-22}$ & $3.32 \times 10^{-14}$ & $l_{P} m_{P}$ & $\hbar$ & $v$ & $v$ \\
\hline 0.5 & $7.72 \times 10^{-13}$ & $4.55 \times 10^{-31}$ & $2.58 \times 10^{-21}$ & $1.37 \times 10^{-22}$ & $1.02 \times 10^{-14}$ & $l_{P} m_{P}$ & $\hbar$ & $v$ & $v$ \\
\hline$\alpha(0.0073)$ & $5.29 \times 10^{-11}$ & $6.65 \times 10^{-33}$ & $1.77 \times 10^{-19}$ & $1.99 \times 10^{-24}$ & $2.18 \times 10^{-18}$ & $l_{P} m_{P}$ & $\hbar$ & $v$ & $v$ \\
\hline
\end{tabular}

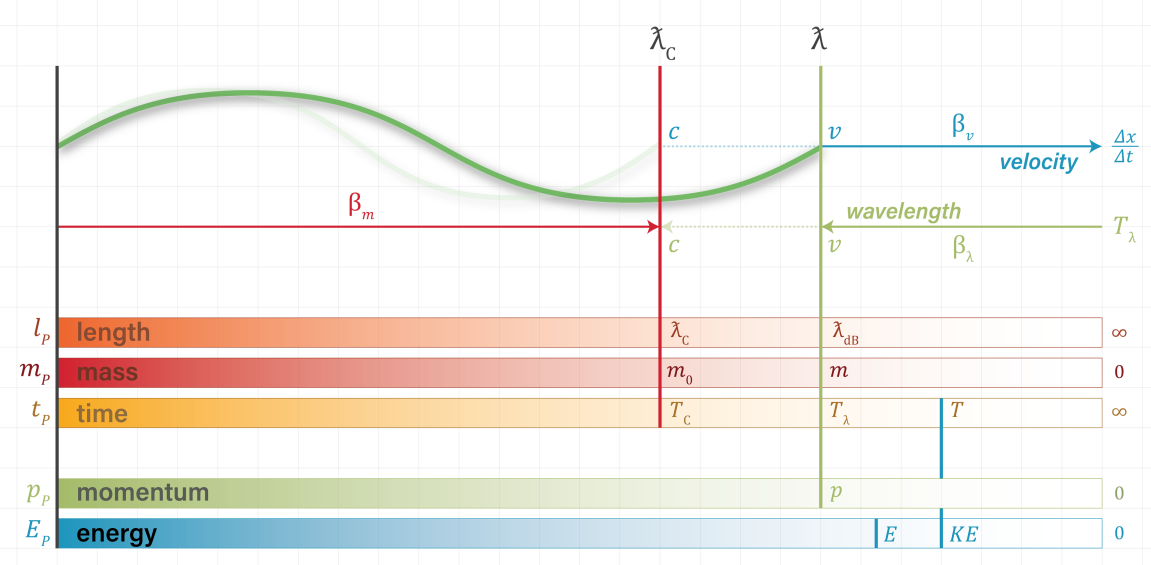

Fig. 8: The basic mechanics of massive particles are illustrated in the relationships between length, mass, and time. The figure applies the rest mass, wavelength, and velocity operators to the maximum Planck potentials. The spin operator is not shown in the figure.

For every value of the wavelength and velocity operators, the quantum constant and Planck's constant are conserved through the spatial and temporal currents $v^{2}$.

Figure 8 is a conceptual illustration of the massive particle mechanics described by the New Foundations Model. As shown in the illustration, the $\beta_{m}$ operator determines the particle's Compton wavelength, which has a dual meaning. It is both the measure of a particle's rest mass in terms of a photon's wavelength, and also the limit of the particle's own wavelength as it approaches the speed of light. The spin operator for particles with rest mass is not shown in the illustration, but reduces the maximum kinetic energy potential by half.

It may seem that rest mass should increase a particle's kinetic energy but that does not appear to be the case. A comparison of the kinetic energy equations for photons and electrons 
shows that kinetic energy in both cases is determined by a particle's wavelength regardless of the presence of rest mass. It is as if the particle's rest energy is just sufficient to overcome Higgs field resistance, after which the same rules of inertial mechanics apply to bosons and fermions.

Given the application of rest mass, the illustration shows that a particle's wavelength is further refined by the $\beta_{\lambda}$ operator, and its velocity is determined by the equivalent $\beta_{v}$ operator. The maximum potential of these operators is shown as $c$ at the Compton wavelength. The operators approach zero as wavelength increases.

The illustration also shows how different quantities of length, mass, and time relate to each other. For a given wavelength there is a corresponding quantity of mass and time, where rest mass is also present but does not affect the kinetic energy. The time unit $T_{\lambda}$ gives the particle's wavelength in units of seconds, normalizing the wavelength and velocity operators as $v^{2} / c^{2}$. The second time component is the particle's oscillation period $T$, which increases with the combined effects of longer wavelength and slower rate of displacement.

\subsection{Demonstration on the Bohr Hydrogen Atom}

The New Foundations Model can be demonstrated on the Bohr hydrogen atom. The electron's properties described by several constants in table 2 are reinterpreted here in terms of maximum potentials and reduction operators.

\subsubsection{Rydberg Energy}

The kinetic energy of a ground state electron is given by the Rydberg energy formula. The Rydberg energy is determined using the Rydberg constant from table 2

$$
\frac{1}{4 \pi}\left(\frac{m_{e}}{m_{p}}\right) \frac{\alpha^{2}}{l_{p}}
$$

where $m_{P}$ is the Planck mass. The Rydberg constant is a collection of operators described in table 7 that transform the Planck energy into the ground state energy of the electron. In the Rydberg constant we find the $\beta_{m}$ operator in the ratio $m_{e} / m_{P}$, the wavelength and velocity operators in the form of $\alpha^{2}$, and a half spin operator. An additional value of $2 \pi$ and the Planck length appear in the denominator.

To determine the ground state energy of the electron, the Rydberg constant is multiplied by $h c$ which we can write in Planck units as

$$
h c=2 \pi l_{P} E_{P} .
$$

Multiplying the Rydberg constant by $h c$ gives

$$
\frac{1}{22 \pi}\left(\frac{m_{e}}{m_{p}}\right) \frac{\alpha^{2}}{l_{p}} 2 \pi E_{P} l_{p} .
$$

The formula shows that a superfluous value of $l_{P}$ is included in both the Rydberg constant and $h c$. Unnecessary quantities are easy to identify and remove when we use the elementary forms of the constants. The unnecessary Planck lengths cancel as does the angular constant $2 \pi$ in the numerator and denominator. The remaining formula

$$
\frac{1}{2}\left(\frac{m_{e}}{m_{p}}\right) \alpha^{2} E_{P}
$$


may be considered the operator form of the kinetic energy formula $E=1 / 2 m v^{2}$, where extensive values of mass and velocity are replaced by proportionality operators acting on the maximum Planck energy potential. We can summarize the operators as

- Rest mass operator: $\beta_{m}\left(m_{e} / m_{P}\right), 4.185 \times 10^{-23}$

- Spin operator: $1 / 2$

- Wavelength operator: $\beta_{\lambda}(\alpha), 0.0073$

- Velocity operator: $\beta_{v}(\alpha), 0.0073$

Applying the operators to the Planck energy shows how the ground state energy is produced

$$
\begin{aligned}
E_{P} & =1.2209 \times 10^{19} \mathrm{GeV} \\
\times 4.185 \times 10^{-23} & =510,999 \mathrm{eV} \\
\times 1 / 2 & =255,500 \mathrm{eV} \\
\times 0.0073^{2} & =13.6 \mathrm{eV}
\end{aligned}
$$

\subsubsection{Bohr Radius}

Bohr proposed calculating the allowed electron orbitals using the electrostatic force [17]

$$
r_{n}=\frac{n^{2} \hbar^{2}}{Z k_{e} e^{2} m_{e}}
$$

where $\mathrm{n}$ is the orbital number and $\mathrm{Z}$ is the integer number of protons in the nucleus. We can simplify Bohr's formula by substituting Planck units for $\hbar, K_{e}$, and $e$ according to the formulas in table 2

which yields

$$
r_{n}=\left(\frac{n^{2}}{Z}\right)\left(\frac{l_{P}^{z} l_{P} m_{P} m_{P}}{t_{P}^{f}}\right)\left(\frac{t_{P}^{*}}{l_{P}^{z} m_{P}}\right)\left(\frac{1}{t_{P}^{\not} \alpha}\right) \frac{1}{m_{e}}
$$

$$
r_{n}=\left(\frac{n^{2}}{Z}\right)\left(\frac{m_{P}}{m_{e}}\right)\left(\frac{1}{\alpha}\right) l_{P} .
$$

Multiplying the inverse rest mass operator $m_{P} / m_{e}$ and the inverse wavelength operator $1 / \alpha$ by the Planck length gives the electron's wavelength, simplifying the formula to

$$
r_{n}=\left(\frac{n^{2}}{Z}\right) \lambda
$$

where $\lambda$ is equal to $5.29 \times 10^{-11} \mathrm{~m}$. The remaining ratio $n^{2} / Z$ accounts for the intervals of different atomic numbers and orbitals. For the ground state of the hydrogen atom, the formula shows that the Bohr radius is equal to the electron's wavelength.

We can show that the de Broglie formula produces the same result as the electrostatic formula by inserting the rest mass and wavelength operators into 6

$$
\lambda=\left(\frac{m_{P}}{m_{0}}\right)\left(\frac{c}{v}\right) l_{p}
$$

The velocity operator $c / v$ is equal to the wavelength operator so we can also write the equation as

$$
\lambda=\left(\frac{m_{P}}{m_{e}}\right)\left(\frac{1}{\alpha}\right) l_{p} .
$$


3.6.3 Electron ground state velocity

Bohr compared the centripetal force of the electron to the electrostatic force [17], giving the equivalence

$$
\frac{m_{e} v^{2}}{r}=\frac{k_{e} e^{2}}{r^{2}}
$$

which can be arranged into a formula for the electron's velocity

$$
v^{2}=\frac{k_{e} e^{2}}{m_{e} r} .
$$

The formula can be written in natural units where the constants $k_{e}$ and $e^{2}$ are replaced by the formulas in table 2 . We can also substitute $\lambda$ for $r$ in the ground state where the radius and wavelength are equal according to 23

$$
\begin{aligned}
v^{2} & =\frac{l_{P} m_{P} c^{2}}{t / P} \frac{t f_{P}^{\prime} \alpha}{t m_{e}} \\
& =\left(\frac{l_{P}}{t}\right)\left(\frac{m_{P}}{m_{e}}\right) \alpha c^{2} .
\end{aligned}
$$

To reduce the formula further, we can replace $l_{P} / \lambda$, which includes the $\beta_{m}$ and $\beta_{\lambda}$ operators, with two equivalent forms. Substituting $m_{e} / m_{P}$ for the rest mass operator and $\alpha$ for the wavelength operator gives

$$
v^{2}=\left(\frac{m_{e}}{m_{P}}\right)\left(\frac{m_{P}}{m_{e}}\right) \alpha^{2} c^{2} .
$$

We now have offsetting rest mass operators that cancel, leaving just the wavelength and velocity operators acting on the maximum velocity $c$

$$
\begin{aligned}
v & =\sqrt{\alpha^{2} c^{2}} \\
& =\alpha c .
\end{aligned}
$$

\subsection{Total Mechanical Energy}

The total mechanical energy of a particle is found by combining its kinetic energy of motion with its latent rest energy. The energy-momentum formula blends these two vectors. The significance of the total energy can be evaluated in terms of the particle's rest and inertial masses. The standard form of the energy-momentum formula

$$
E=\sqrt{(p c)^{2}+\left(m_{0} c^{2}\right)^{2}}
$$

can be restated in terms of mass as

$$
E=\sqrt{m^{2}+m_{0}^{2}} c^{2}
$$

where $m$ is the inertial mass and $m_{0}$ is the rest mass.

In this form we can evaluate the two mass vectors while treating $c^{2}$ as a unit conversion. The result of the formula is to blend a fixed quantity of rest mass with a variable quantity of inertial mass. 
It is worth considering whether dimensions of $M L T^{-1}$ are the best choice for quantifying momentum, given that these units were chosen without a knowledge of matter's wave-like properties. We could simplify mechanical equations using inertial mass, applying a dimensionless wavelength operator $\lambda_{C} / \lambda$ to the rest mass. This would use the inverse mass-wavelength relationship to quantify the effects of inertia in units of kilograms. Multiplying by velocity introduces motion into the formula in dimensions of length per time, and Energy would be quantified in $\mathrm{kgm} / \mathrm{s}$. This approach removes the confusing use of time to quantify the particle's wavelength.

We could go even further in treating mass and energy as equivalent. Replacing the extensive value of velocity with its operator form, $v / c$, we could quantify energy in units of $\mathrm{kg}$. Quantities of mass, momentum, and energy would all be calculated using only the Planck mass and dimensionless operators on a scale of 0 to 1 . This would give the following three, simple equations for calculating energy. The first formula applies to particles without rest mass, the second formula gives the kinetic energy of particles with rest mass, and the third formula gives the total energy of particles with rest mass-each quantified in $\mathrm{kg}$

$$
\begin{aligned}
E_{\gamma} & =m_{P}\left(\frac{l_{P}}{\lambda}\right) \\
K E & =m_{P}\left(\frac{l_{P}}{\lambda}\right)\left(\frac{v}{c}\right)\left(\frac{1}{2}\right) \\
E & =m_{P}\left(\frac{l_{P}}{\lambda}\right) \sqrt{1+\left(\frac{\lambda}{\lambda_{C}}\right)^{2}} .
\end{aligned}
$$

I'm not advocating a change in unit system so much as I'm casting light on the physical meanings of mass and momentum, and their use in formulas to describe particle mechanics.

From this simplified form of the total energy equation we can create an operator specifically for calculating a particle's total energy

$$
\beta_{E}=\sqrt{1+\left(\frac{\lambda}{\lambda_{C}}\right)^{2}}
$$

where total energy is calculated as $\beta_{m} \beta_{\lambda} \beta_{E} E_{P}$

An interesting question is whether the full Planck energy potential $E_{P}$ is conserved somehow by each particle. In the case of a photon, the proportionality operator is such that for any length photon, the Planck energy can be evenly distributed in intervals of the Planck length across its wavelength. One implication might be that the reduced quantity of energy encountered in a photon interaction is actually present at each interval of the photon's length, but only interacts at one location. Determining where the photon will interact can only be answered by resolving the measurement problem.

The same question could be asked about particles with rest mass, where rest mass can be evenly distributed across the Compton wavelength and further diluted by the wavelength and velocity operators according to its velocity. We can imagine a fixed quantity of energy being conserved across space and time as the driving force behind particle mechanics. From slowly moving particles with long wavelengths to high speed particles with shorter wavelengthsand everything in between - the Planck energy may be conserved by the proposed operators while lending different quantities of energy to interactions between particles. 


\subsubsection{Demonstrating the model in natural units}

Table 9 demonstrates the proposed operators and equations using two scenarios. The first scenario treats the Planck units as natural units of length, mass, and time, where units of length are integer multiples of the Planck length. The example demonstrates in simple ratios the physical transformations made by each operator and equation. The second example calculates the same properties for a ground state electron in the hydrogen atom.

Table 9: The New Foundations Model is summarized in two examples: one example in natural units, and another example of the ground state electron.

\begin{tabular}{|c|c|c|c|c|c|}
\hline Name & Symbol & Operator form & Formula & e.g. natural units & e.g. H1 $1 s$ electron \\
\hline Planck length & $l_{P}$ & - & - & 1 & $1.616255 \times 10^{-35} \mathrm{~m}$ \\
\hline Planck mass & $m_{P}$ & - & - & 1 & $2.176434 \times 10^{-8} \mathrm{~kg}$ \\
\hline Planck time & $t_{P}$ & - & - & 1 & $5.391247 \times 10^{-44} s$ \\
\hline Rest mass operator & $\beta_{m}$ & - & $\frac{m_{0}}{m_{P}}, \frac{l_{P}}{\lambda_{C}}$ & $1 / 10$ & $4.185463 \times 10^{-23}$ \\
\hline Wavelength operator & $\beta_{\lambda}$ & - & $\frac{\lambda_{C}}{\lambda}, \frac{m}{m_{0}}$ & $10 / 25$ & 0.007297353 \\
\hline Velocity operator & $\beta_{v}$ & - & $\frac{\lambda t_{P}}{T l_{P}}, \frac{v}{c}$ & $10 / 25$ & 0.007297353 \\
\hline Spin operator & $s$ & - & $\frac{1}{2}$ & $1 / 2$ & 0.5 \\
\hline Total energy operator & $\beta_{E}$ & - & $\sqrt{1+\left(\frac{\lambda}{\lambda_{C}}\right)^{2}}$ & $\sqrt{\left(1+(25 / 10)^{2}\right.}$ & 137.0396 \\
\hline Compton wavelength & $\lambda_{C}$ & $\frac{l_{P}}{\beta_{m}}$ & $\frac{l_{P} m_{P}}{m_{0}}$ & 10 & $3.861593 \times 10^{-13} \mathrm{~m}$ \\
\hline Wavelength & $\lambda$ & $\frac{l_{P}}{\beta_{m} \beta_{\lambda}}$ & $\frac{l_{P} m_{P}}{m}$ & 25 & $5.291772 \times 10^{-11} \mathrm{~m}$ \\
\hline Rest mass & $m_{0}$ & $m_{P} \beta_{m}$ & $\frac{l_{P} m_{P}}{\lambda_{C}}$ & $1 / 10$ & $9.109384 \times 10^{-31} \mathrm{~kg}$ \\
\hline Inertial mass & $m$ & $m_{P} \beta_{m} \beta_{\lambda}$ & $\frac{l_{P} m_{P}}{\lambda}$ & $1 / 25$ & $6.647438 \times 10^{-33} \mathrm{~kg}$ \\
\hline T Lambda & $T_{\lambda}$ & $\frac{t_{P}}{\beta_{m} \beta_{\lambda}}$ & $\frac{\lambda}{c}$ & 25 & $1.765145 \times 10^{-19} s$ \\
\hline Momentum & $p$ & $p_{P} \beta_{m} \beta_{\lambda}$ & $\frac{m_{0} \lambda_{C}}{T_{\lambda}}, \frac{m \lambda}{T_{\lambda}}$ & $1 / 25$ & $1.992852 \times 10^{-24} \mathrm{kgm} / \mathrm{s}$ \\
\hline Velocity & $v$ & $\frac{l_{P}}{t_{P}} \beta_{v}$ & $\frac{t}{T}, \frac{\Delta x}{\Delta t}$ & $25 / 62.5$ & $2,187,691 \mathrm{~m} / \mathrm{s}$ \\
\hline Period & $T$ & $\frac{t_{P}}{\beta_{\lambda}} \beta_{v}$ & $\frac{T_{\lambda} \lambda}{\lambda_{C}}$ & $25^{2} / 10$ & $2.418884 \times 10^{-17} s$ \\
\hline Kinetic Energy & $K E$ & $\beta_{m} \beta_{\lambda} \beta_{v} s E_{P}$ & $\frac{m \hbar^{2}}{2 T_{\lambda} T}$ & $\frac{(1 / 25)\left(25^{2}\right)}{(2)(25)(62.5)}$ & $2.179872 \times 10^{-18} \mathrm{~J}$ \\
\hline Total Energy & $E$ & $\beta_{m} \beta_{\lambda} \beta_{E} E_{P}$ & $\sqrt{m^{2}+m_{0}^{2}} c^{2}$ & $\sqrt{(1 / 25)^{2}+(1 / 10)^{2}} 1^{2}$ & $8.187324 \times 10^{-14} J$ \\
\hline
\end{tabular}

The simplicity of elementary particle mechanics — under isolated conditions — can be demonstrated with the mechanical operators. We can create a consolidated operator $\beta$ as the 
product of the four kinetic energy operators

$$
\beta=\frac{1}{2}\left(\frac{l_{P}}{\lambda_{C}}\right)\left(\frac{\lambda_{C}}{\lambda}\right)\left(\frac{\lambda}{T} \frac{t_{P}}{l_{P}}\right)
$$

that reduces to a new time operator multiplied by the spin operator

$$
\beta_{t} \beta_{s}=\frac{t_{P}}{2 T}
$$

This single, dimensionless operation applied to the Planck energy gives the particle's kinetic energy

$$
K E=\beta_{t} \beta_{s} E_{P}
$$

while removing the spin operator gives the correct value of photon energy.

The simplicity of this formula reflects the power of natural symmetries in constraining the possible quantities of a particle's mechanical properties. Given nothing more than its temporal period, we can determine the particle's wavelength, mass, momentum, and energy. The symmetries in table 6 and the maximum Planck potentials make this possible. The same result can be calculated using the traditional formula $\hbar / 2 T$, but deriving the formula from the operators gives a physical description of the transformations that we don't get from Planck's constant. The time operator depicts a maximum Planck energy diluted over the particle's oscillation period.

We can summarize the time operator as the product of mass, wavelength, and velocity operators

$$
\beta_{t}=\beta_{m} \beta_{\lambda} \beta_{v}
$$

\subsubsection{Action}

The principle of action uses the constraints imposed by fundamental symmetries to predict particle trajectories. We can derive the formulas for action by multiplying the combined dimensionless operator presented in the previous section by the Planck energy. Expanding the formula gives

$$
K E=\frac{1}{2}\left(\frac{l_{P}}{\partial_{C}}\right)\left(\frac{\not_{C}}{\not t}\right)\left(\frac{\not}{T} \frac{t_{P}}{l_{P}}\right) m_{P}\left(\frac{l_{P}}{t_{P}}\right)\left(\frac{l_{P}}{t_{P}}\right)
$$

which reduces to the following equality, presented earlier as equation 20 where $l_{P} P_{P}$ can be any combination of $\lambda p$

$$
T E_{k}=\frac{l_{P} p_{P}}{2} .
$$

$T$ is the oscillation period and $K E$ is the kinetic energy. Additional equations of action can be created using combinations of terms conserving $\hbar$, including $l_{P} p_{P}, E_{P} t_{P}, \lambda p$, and $T E$.

\section{Gravity}

An important pursuit in modern day physics is a theory that unites quantum mechanics with general relativity. The new form of the gravitational constant in 2 along with the principles of maximum potential and proportionality may offer clues for uniting the theories. The gravitational constant shows that maximum gravitational potentials are quantified in the same units used by particle mechanics, and proportionality operators are also constructed from the ratios of length, mass, and time. 
The gravitational function is expressed in three parts

$$
G=\left(\frac{\mathbf{l}_{\mathbf{P}}}{\mathbf{m}_{\mathbf{P}}}\right)\left(\frac{\mathbf{l}_{\mathbf{P}}}{\mathbf{t}_{\mathbf{P}}}\right)\left(\frac{\mathbf{l}_{\mathbf{P}}}{\mathbf{t}_{\mathbf{P}}}\right)
$$

The first component is the ratio of Planck length to Planck mass, giving an inverse linear mass density in units of $\mathrm{m} / \mathrm{kg}$. The quotient of these two Planck units produces the principal maximum potential for gravitational bodies, equal to $7.43 \times 10^{-28} \mathrm{~m} / \mathrm{kg}$. The significance of this ratio is seen in the attributes of black holes where the relationship between the Schwarzschild radius and the mass is always $7.43 \times 10^{-28} \mathrm{~m} / \mathrm{kg}$, according to equation 7 . A limit on black hole linear density may suggest that black holes are not singularities, and that matter engulfed by a black hole cannot be compressed beyond this limit.

The properties of existing and theoretical gravitational bodies are given in table 10, where the properties of theoretical bodies are determined from the equations.

Table 10: Attributes of existing and theoretical gravitational bodies are shown, including black holes and other less dense bodies

\begin{tabular}{lrrrrr}
\hline Body & Mass $(\mathrm{kg})$ & Radius $(\mathrm{m})$ & Mass density $(\mathrm{m} / \mathrm{kg})$ & Velocity $(\mathrm{m} / \mathrm{s})$ & Accel $\left(\mathrm{m} / \mathrm{s}^{2}\right)$ \\
\hline Planck mass bh & $2.1764 \times 10^{-8}$ & $3.2325 \times 10^{-35}$ & $7.4262 \times 10^{-28}$ & $299,792,458$ & $1.3920 \times 10^{51}$ \\
Sagittarius A* & $8.2582 \times 10^{36}$ & $1.2265 \times 10^{10}$ & $7.4262 \times 10^{-28}$ & $299,792,458$ & $3.6638 \times 10^{6}$ \\
Black hole Sun & $1.9885 \times 10^{30}$ & $2.9533 \times 10^{3}$ & $7.4262 \times 10^{-28}$ & $299,792,458$ & $1.5216 \times 10^{13}$ \\
Sun & $1.9885 \times 10^{30}$ & $6.9570 \times 10^{8}$ & $1.7493 \times 10^{-22}$ & 617,684 & 274.21 \\
Black hole Earth & $5.9723 \times 10^{24}$ & $8.8703 \times 10^{-3}$ & $7.4262 \times 10^{-28}$ & $299,792,458$ & $5.0661 \times 10^{18}$ \\
Earth & $5.9723 \times 10^{24}$ & $6.3781 \times 10^{6}$ & $5.3398 \times 10^{-19}$ & 11,180 & 9.7985 \\
\hline
\end{tabular}

The signature characteristics of gravitation equations are inputs of mass in the numerator and length in the denominator. The gravitational function uses these inputs as proportionality operators against the maximum linear mass density $l_{P} / m_{P}$. The combination of these two inputs with the maximum potential in the function gives the principal operator $\beta_{\rho}$, signifying the intensive relationship between a body's radius and its mass

$$
\beta_{\rho}=\left(\frac{l_{P}}{l}\right)\left(\frac{M}{m_{P}}\right)
$$

where $l$ is the radius of the gravitational body and $M$ is its mass. Figure 9 illustrates the relationship between length and mass as it pertains to gravitational bodies. The maximum density $7.43 \times 10^{-28}$ is plotted at 45 degrees from the origin, signifying a 1:1 relationship between length and mass in natural units. According to the Schwarzschild radius formula, all black holes have the same linear mass density as this determines the horizon of the black hole by its mass. Black holes are only separated along this line by extensive quantities of their masses and radii.

No gravitational bodies appear above the line as the limit on linear mass density appears to forbid it. Bodies with less than the maximum density fall below the line according to their densities.

The linear mass density operator works at arbitrary distances from the center of a body including its horizon and locations above. The role of linear mass density in determining gravitational potential can be shown in the formula for velocity potential. 


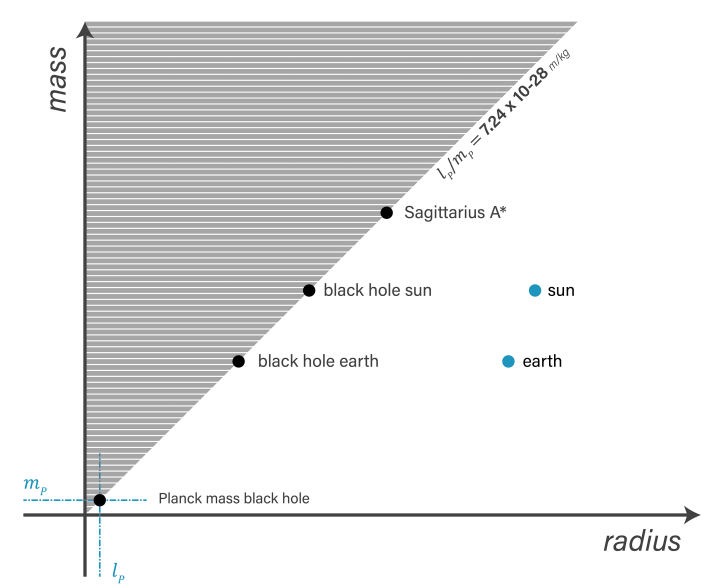

Fig. 9: The maximum linear mass density of black holes is the constant $l_{P} / m_{P}$

\subsection{Velocity}

The traditional formula for escape velocity at a distance $r$ from the center of a gravitational body

$$
v_{e}^{2}=\frac{2 G M}{r}
$$

can be re-stated as

$$
v_{e}^{2}=2\left(\frac{l_{P}}{m_{P}} c^{2}\right) \frac{M}{r} .
$$

Re-arranging the terms demonstrates the $\beta_{\rho}$ operator which acts on two instances of $c$

$$
v_{e}=\sqrt{2\left(\frac{M}{m_{P}}\right)\left(\frac{l_{P}}{r}\right) c^{2}} .
$$

The simplified formula for escape velocity is

$$
v_{e}=\sqrt{2 \beta_{\rho}} c .
$$

The formula shows that selecting the Schwarzschild radius as the distance r produces an escape velocity of $c$ at the horizon.

The second and third components of the gravitational function are two instances of $c$, the maximum velocity potential. If we add an input for the mass of a body at a distance $\mathrm{r}$, we get a formula for energy in units of $\mathrm{kgm}^{2} / \mathrm{s}^{2}$.

\subsection{Energy}

The formula for gravitational potential energy

$$
U_{g}=-G \frac{M m}{r}
$$


can be restated in Planck units as

$$
U_{g}=-\left(\frac{l_{P}}{r}\right)\left(\frac{M}{m_{P}}\right) m c^{2} .
$$

The mass of the second body can also be represented as $m m_{P} / m_{P}$, allowing us to state the equation in terms of energy potential as

$$
U_{g}=-\left(\frac{l_{P}}{r}\right)\left(\frac{M}{m_{P}}\right)\left(\frac{m}{m_{P}}\right) E_{P}
$$

or simply

$$
U_{g}=-\beta_{\rho} \beta_{m} E_{P} .
$$

According to the energy and velocity equations, the $\beta_{\rho}$ operator is the right reduction for the gravitational field to transform two values of $c$ into the wavelength and velocity operators described by the New Foundations Model. The mass $m$ is already entered in reduced form so the $\beta_{\rho}$ operator is applied entirely to $c^{2}$.

It is worth calling out this correspondence between mechanical and gravitational functions. The gravitational function determines the strength of gravitational potentials in the same terms we use to describe the mechanical properties of elementary particles. The proportionality operator $\beta_{\rho}$ produces a field strength equal to the mechanical strength of a particle determined by its wavelength and velocity operators. The conservation of masswavelength proposed in 3.2 makes this possible.

Revisiting the formula for escape velocity, we see that taking the square root of the linear mass density operator gives the value for each of the wavelength and velocity operators. Individual proportions of length and mass do not need to be the same as long as we take the square root of the product of length and mass.

The application of the $\beta_{\rho}$ operator on $c^{2}$ tells us something important about the natural intervals and ratios of length, mass, and time. We saw with particle mechanics that applying a length operator to the maximum mass potential gives the correct quantity of mass. Now we also see from the linear mass density operator that we can apply a ratio of length/mass onto maximum units of length/time and get the correct velocity.

To produce values of acceleration and force, the gravitational function requires an additional operator in the ratio of Planck length to distance $\mathrm{r}$. We can call this operator $\beta_{l}$ for the reduction in length potential.

\subsection{Acceleration}

The traditional formula for gravitational acceleration potential

$$
g=-\frac{G M}{r^{2}}
$$

can be re-written as

$$
g=-\left(\frac{l_{P}}{m_{P}} c^{2}\right) \frac{M}{r^{2}} .
$$

Arranging the equation into operators and maximum potentials gives

$$
g=-\left(\frac{M}{m_{P}}\right)\left(\frac{l_{P}}{r}\right)\left(\frac{l_{P}}{r}\right)\left(\frac{l_{P}}{t_{P}^{2}}\right) .
$$


The formula shows that the acceleration is given by the usual linear mass density operator combined with the dimensionless length operator in the ratio of $l_{P} / l$. What remains is the maximum acceleration potential in units of $\mathrm{m} / \mathrm{s}^{2}$

$$
=-\beta_{\rho} \beta_{l} a_{P}
$$

where $a_{P}$ is the maximum acceleration potential.

\subsection{Force}

The traditional formula for gravitational force potential

$$
F=-G \frac{M m}{r^{2}}
$$

can be expressed by the gravitational function as

$$
F=-\left(\frac{M}{m_{P}}\right)\left(\frac{l_{P}}{r}\right)\left(\frac{l_{P}}{r}\right) m a_{P} .
$$

The mass of the second body can also be represented as $m m_{P} / m_{P}$, allowing us to state the equation in terms of force potential as

$$
F=-\left(\frac{M}{m_{P}}\right)\left(\frac{l_{P}}{r}\right)\left(\frac{l_{P}}{r}\right)\left(\frac{m}{m_{P}}\right) F_{P}
$$

which simplifies to the following set of operators

$$
F=-\left(\beta_{\rho} \beta_{l} \beta_{m}\right) F_{P}
$$

Because the mass is entered in reduced form, the two operators are only applied to the maximum acceleration potential. The product of mass and acceleration gives the force.

Even the complex Einstein Field Equations describe force in terms of maximum potentials. The right-hand side of the Einstein Field Equations

$$
\frac{8 \pi G}{c^{4}} T_{u v}
$$

can be restated as a maximum force potential

$$
8 \pi\left(\frac{t_{P}}{p_{P}}\right) T_{u v}
$$

where $t_{P} / p_{P}$ is the inverse Planck force-a quantity that appears in many of the constants in table 2. In this case the stress energy tensor acts as an operator on the maximum force potential to characterize the mass-energy content of spacetime.

Table 11 summaries the gravitational functions and operators. 
Table 11: A summary of operators used by the gravitational function. The function calculates physical quantities by applying proportionality operators to maximum potentials embedded in the function.

\begin{tabular}{rlrr}
\hline Property & Operators & Simple Function & Traditional function \\
\hline Velocity & $\beta_{\rho}$ & $v=-\sqrt{2 \beta_{\rho}} c$ & $v_{e}=-\sqrt{\frac{2 G M}{r}}$ \\
Energy & $\beta_{\rho}, \beta_{m}$ & $E=-\beta_{\rho} \beta_{m} E_{P}$ & $U_{g}=-\frac{G M m}{r}$ \\
Acceleration & $\beta_{\rho}, \beta_{l}$ & $a=-\beta_{\rho} \beta_{l} a_{P}$ & $g=-\frac{G M}{r^{2}}$ \\
Force & $\beta_{\rho}, \beta_{l}, \beta_{m}$ & $f=-\beta_{\rho} \beta_{l} \beta_{m} F_{P}$ & $F=-\frac{G M m}{r^{2}}$ \\
\hline
\end{tabular}

\section{Electromagnetism}

The New Foundations Model explains electromagnetic potential using the same principles that describe particle mechanics and gravitational potential. The electromagnetic constants are also maximum potentials comprising the Planck length, mass, and time; and proportionality operators determine the electromagnetic field potentials. These similarities are hidden by the unique unit types quantifying the electromagnetic interaction. With the conversion of electromagnetic constants into Planck units in table 2, the electromagnetic interaction can now be interpreted entirely in units of length, mass, and time. Table 12 gives a conversion for each electromagnetic unit into MKS units.

Table 12: Electromagnetic units have equivalent values in kilograms, meters, and seconds. The table provides a conversion factor for each unit type derived from the Planck unit definitions of the electromagnetic constants.

\begin{tabular}{rlllr}
\hline Em Unit & Symbol & Formula & Conversion & MKS units \\
\hline coulomb & q, e & $q_{P} / q_{P}$ & $2.874495 \times 10^{-26}$ & $\mathrm{~s}$ \\
ampere & $\mathrm{A}$ & $I_{P} / I_{P}$ & $2.874495 \times 10^{-26}$ & dimensionless \\
volt & $\mathrm{V}$ & $V_{P} / V_{P}$ & $3.478872 \times 10^{25}$ & $\mathrm{kgm}^{2} / \mathrm{s}^{3}$ \\
tesla & $\mathrm{T}$ & $B_{P} / B_{P}$ & $3.478872 \times 10^{25}$ & $\mathrm{~kg} / \mathrm{s}^{2}$ \\
weber & $\mathrm{Wb}$ & $V_{s}$ & $3.478871 \times 10^{25}$ & $\mathrm{kgm}^{2} / \mathrm{s}^{2}$ \\
ohm & $\Omega$ & $Z_{P} / Z_{P}$ & $1.210255 \times 10^{51}$ & $\mathrm{kgm}^{2} / \mathrm{s}^{3}$ \\
henry & $\mathrm{H}$ & $L_{P} / L_{P}$ & $1.210255 \times 10^{51}$ & $\mathrm{kgm}^{2} / \mathrm{s}^{2}$ \\
farad & $\mathrm{F}$ & $C_{P} / C_{P}$ & $8.262723 \times 10^{-52}$ & $\mathrm{~s}^{4} / \mathrm{kgm}^{2}$ \\
siemen & $\mathrm{S}$ & $G_{P} / G_{P}$ & $8.262720 \times 10^{-52}$ & $\mathrm{~s}^{3} / \mathrm{kgm}^{2}$ \\
\hline
\end{tabular}

Conversion factors were calculated as the ratio of the MKS value to the traditional value, using the constants noted in the table.

The principal function of the electromagnetic interaction is Coulomb's law, given by the Coulomb constant and the inverse electric permittivity. Like the gravitational constant, Coulomb's constant is comprised of fundamental Planck units. The constant includes eight 
units, simplified below into six where Planck momentum replaces $l_{P} m_{P} / t_{P}$

$$
\text { force }=\left(\frac{\mathbf{p}_{\mathbf{P}}}{\mathbf{t}_{\mathbf{P}}}\right)\left(\frac{\mathbf{l}_{\mathbf{P}}}{\mathbf{t}_{\mathbf{P}}}\right)\left(\frac{\mathbf{l}_{\mathbf{P}}}{\mathbf{t}_{\mathbf{P}}}\right) .
$$

Coulomb's law gives the forces of attraction and repulsion due to the electromagnetic interaction. The function converts inputs of charge and distance into units of force, where the maximum force potential $p_{P} / t_{P}$ is embedded inside the function. To measure the force between two locations, the function requires two operators for each location. The first operator is the ratio of charge to Planck time, where charge is a rate of change quantified in seconds. While charge is traditionally quantified in units of coulomb, the model shows that electric charge is naturally quantified in units of time.

The second operator is the ratio of Planck length to the distance between locations, given in meters. The four total inputs-two from each location-have dimensions of $s^{2} / \mathrm{m}^{2}$, which pair up with the embedded value of $c^{2}$ to create dimensionless operators. The operators act on the maximum Planck force potential also embedded in the constant.

The inverse square relation found in the strengths of gravitational and electromagnetic potentials reflect the 2-part mechanism determining particle wavelength and velocity as described in section 3.4.1. Electromagnetic and gravitational potentials might be thought of as stretching and compressing particle oscillations, affecting their wavelengths and velocities in equal measure.

Unfortunately, the Planck units do not reveal what physical attribute distinguishes one type of electric charge from another. For now, the Planck unit formulation, like other descriptions of electromagnetism, can only say what electric charge does and not what it is. Perhaps a new formulation of electromagnetism in MKS units will lead to a physical description like the zitterbewegung motion of particles to explain electric charge [18].

According to the formulas in table 2, the maximum charge potential is the Planck charge. The elementary charge is related to the Planck charge according to the formula

$$
e=t_{P} \sqrt{\alpha} \text {. }
$$

At a glance, it might appear as though the elementary charge of $4.6 \times 10^{-45} s$ is a shorter time duration than the Planck time. Closer examination shows, however, that the square root of the fine structure constant embedded in the elementary charge increases the time input paired with the Planck time, effectively reducing the potential and not increasing it.

In light of the Planck unit formulas, we can treat the elementary charge as applying a standard proportionality operator to the maximum Planck charge. The amount of reduction between charges is the fine structure constant, divided equally between two instances of the elementary charge.

Another important constant defined in Planck units is the Planck current

$$
I_{P}=\frac{t_{P}}{t_{P}}
$$

The Planck current is a dimensionless ratio of Planck time to Planck time in MKS units, reflecting the net difference between charges. In MKS units, we see how a dimensionless current describes the relationship between potential and resistance. Ohm's law

$$
V=I R
$$

can be written in Planck units as

$$
\frac{E_{P}}{t_{P}}=\left(\frac{t_{P}}{t_{P}}\right) \frac{E_{P}}{t_{P}}
$$

where the maximum voltage potential and impedance potential are $E_{P} / t_{P}$. 


\section{Comparing Forces}

The New Foundations Model described in sections 3, 4, and 5 provides a method for explaining the similarities and differences in mechanical, electromagnetic, and gravitational forces. Table 13 compares the three different forces under the same scenario of an electron in the hydrogen ground state.

The mechanical force of the electron is compared with the electrostatic and gravitational potentials between the electron and proton. The table begins with the maximum force potential in all three columns and then applies the proper reduction operators to produce the known forces. I use the ground state because the electron's wavelength and radius are equal, making the mechanical and electromagnetic forces comparable. The comparison thus presumes that the electron's wavelength is analogous to the radial distance between electron and proton.

Table 13: Reduction operators for calculating the mechanical, electrostatic, and gravitational forces are shown. Mechanical and electromagnetic forces are equal when particle wavelength is equal to the distance between charges. Gravity is reduced by mass and distance operators.

\begin{tabular}{|c|c|c|c|c|c|}
\hline Reduction & Operator(s) & Formula & Mechanical & Electrostatic & Gravitational \\
\hline Planck Force & $\mathbf{f}_{\mathbf{P}}$ & & $1.21 \times 10^{44}$ & $1.21 \times 10^{44}$ & $1.21 \times 10^{44}$ \\
\hline Electron rest mass & $\beta_{m}$ & $m_{0} / m_{P}$ & $4.19 \times 10^{-23}$ & & $4.19 \times 10^{-23}$ \\
\hline Proton rest mass & $\beta_{m}$ & $m_{0} / m_{P}$ & & & $7.69 \times 10^{-20}$ \\
\hline Electron inertial mass & $\beta_{\lambda}$ & $m_{0} / m$ & $7.30 \times 10^{-3}$ & & \\
\hline Electron period & $\beta_{m}, \beta_{\lambda}, \beta_{v}$ & $T_{\lambda} \lambda / \lambda_{C}$ & $2.23 \times 10^{-27}$ & & \\
\hline Distance & $\beta_{l}$ & $l_{P} / r$ & & $3.05 \times 10^{-25}$ & $3.05 \times 10^{-25}$ \\
\hline Distance & $\beta_{l}$ & $l_{P} / r$ & & $3.05 \times 10^{-25}$ & $3.05 \times 10^{-25}$ \\
\hline Electron charge & $\beta_{q}$ & $t_{P} / t_{P} \sqrt{\alpha}$ & & $8.54 \times 10^{-02}$ & \\
\hline Proton charge & $\beta_{q}$ & $t_{P} / t_{P} \sqrt{\alpha}$ & & $8.54 \times 10^{-02}$ & \\
\hline Total Reductions & & & $6.81 \times 10^{-52}$ & $6.81 \times 10^{-52}$ & $3.00 \times 10^{-91}$ \\
\hline Force & & & $8.24 \times 10^{-8}$ & $8.24 \times 10^{-8}$ & $3.63 \times 10^{-47}$ \\
\hline
\end{tabular}

From the proportionality operators used in the gravitational and electrostatic functions it becomes evident why we experience a weaker force of gravity than electromagnetism. The maximum potential of both interactions is the Planck force which is embedded in the Coulomb and gravitational constants; and both interactions share the same reductions in distance between the proton and electron. What remains explains the difference in electromagnetic and gravitational potentials. The electrostatic force is only further reduced in magnitude by the fine structure constant in the ratio of elementary charge to Planck charge, defined as $t_{P}^{2} \alpha / t_{P}^{2}$. The gravitational potential, on the other hand, is also reduced by the ratios of the particle masses to their maximum mass potentials. The comparable reductions are about 0.7 percent in electric charge versus about 42 orders of magnitude in mass. The effect of rest mass is to substantially reduce the energy potential of massive particles from the maximum energy potential $E_{P}$. If massive particles reached the maximum mass/momentum/energy po- 
tential at the Planck scale, there would be no reduction in the strength of gravity due to these operators.

In physical terms, the mass and energy of a massive particle is limited by its Compton wavelength, which is the maximum limit of its oscillation frequency. The Higgs field that generates rest mass prevents the particle from oscillating faster, reducing its wavelength and increasing its mass. Mass reduction prevents particles from having a larger impact on the gravitational field.

The electromagnetic and gravitational forces in table 13 were calculated using the gravitational constant and coulomb constant functions described in the previous two sections. The mechanical force was also calculated by applying reduction operators to the maximum force potential according to 17

$$
F_{P}=\frac{l_{P} m_{P}}{t_{P}^{2}} \mathrm{kgm} / \mathrm{s}^{2}
$$

To determine which reduction operators to use, we can compare the Planck potential equation to the same formula in reduced quantities of length, mass, and time

$$
F=\frac{\lambda m}{T_{\lambda} T} \mathrm{kgm} / \mathrm{s}^{2}
$$

According to 10 , the value in the numerator is invariant and requires no reduction. We also saw in section 3.7.2 that inputs of wavelength and mass are not necessary for calculating a particle's energy for the same reason.

The denominator has two values of Planck time that $d o$ need to be reduced into values of $T_{\lambda}$ and $T$. These transformations require the following operators:

$$
\begin{aligned}
T_{\lambda} & =t_{P} \beta_{m} \beta_{\lambda} \\
T & =t_{P} \beta_{m} \beta_{\lambda} \beta_{v} .
\end{aligned}
$$

$T_{\lambda}$ transforms the invariant quantum constant $l_{P} m_{P}$ into the right amount of inertial mass, or momentum. The physical property quantified by $T_{\lambda}$ is the spatial reduction from the Planck scale, represented by the particle's wavelength. The mass and wavelength operators produce the correct spatial reduction.

The physical property quantified by $T$ is the particle's temporal reduction from the Planck scale. The mass, wavelength, and velocity operators produce the correct temporal reduction. Table 13 consolidates the five operators into three for comparison with the other forces.

\subsection{Quantized Angular Momentum}

The relative magnitudes of mechanical and electrostatic forces acting on an electron in the hydrogen atom could offer clues about the quantization of momentum and allowed atomic orbitals. The generally accepted formula for angular momentum

$$
L=m_{0} v r_{n}=n \hbar
$$

can be evaluated in detail by replacing $\hbar$ with $l_{P} m_{P} c$

$$
r_{n}=n\left(\frac{m_{P}}{m_{e}}\right)\left(\frac{c}{v}\right) l_{P} .
$$


The formula now resembles the de Broglie relation but with $r_{n}$ replacing wavelength in the ground state. An additional value of the orbital number $n$ appears on the right-hand side accounting for different orbitals.

We can reduce the equation by replacing the rest mass operator and Planck length with the Compton wavelength, and replacing the velocity operator $c / v$ with $n / \alpha$ giving

$$
r_{n}=\lambda_{C}\left(\frac{n^{2}}{\alpha}\right) .
$$

The relationship between radius and wavelength is thus

$$
r_{n}=\lambda_{n} n=a_{0} n^{2}
$$

where $\lambda_{n}$ is the electron wavelength in the $n^{\text {th }}$ orbital, and $a_{0}$ is the Bohr radius.

The formula shows that the electron's wavelength increases in units of $n$ while the radius increases in units of $n^{2}$. However, there is nothing in $\hbar$ that suggests the atomic orbitals are quantized by Planck's constant. As I argued in 3, Planck's constant is invariant for all quantities of $\lambda p$ and $E T$, including for fine and hyperfine transitions of the electron.

We can evaluate the standing wave requirement in light of the mechanical and electrostatic forces acting on the electron. When we compare the relative strengths of the two forces, we find that they balance only in the observed orbitals. This is because moving outward from the ground state, the electrostatic force drops off faster than the mechanical force due to 28 - until the electron wavelength doubles and the two forces balance again. The transition energy is the correct amount to relocate the electron from one orbital to another.

Table 14 gives the energy equivalence of the electromagnetic and mechanical forces for each of the transitions. The change in energy potential due to the electrostatic force was

Table 14: Electrostatic and mechanical forces balance in orbital intervals but not in between.

\begin{tabular}{lccrr}
\hline Trans & $\Delta U(N)$ & $\Delta U(J)$ & $\Delta K E\left(G e / V^{2}\right)$ & $\gamma\left(G e / V^{2}\right)$ \\
\hline $1-2$ & $-7.7196 \times 10^{-8}$ & -10.1987 & -10.1987 & 10.1988 \\
$1-3$ & $-8.1326 \times 10^{-8}$ & -12.0874 & -12.0874 & 12.0875 \\
$1-4$ & $-8.2021 \times 10^{-8}$ & -12.7484 & -12.7484 & 12.7485 \\
$2-3$ & $-4.1298 \times 10^{-9}$ & -1.88865 & -1.88865 & 1.88867 \\
$2-4$ & $-4.8247 \times 10^{-9}$ & -2.54968 & -2.54968 & 2.54970 \\
$3-4$ & $-6.9492 \times 10^{-10}$ & -0.661028 & -0.661028 & 0.661034 \\
\hline
\end{tabular}

calculated by converting force into energy according to 16 .

\section{Fine Tuning the Constants}

Physical constants are comprised of natural quantities of length, mass, and time. It is therefore advantageous to obtain the most accurate values possible for these fundamental units.

The New Foundations Model provides a framework for improving the values of physical constants in table 2 and the Planck units they embody. With the understanding that many traditional constants are comprised of the Planck units, measured values of constants like the speed of light and Planck's constant serve as accurate measurements of the ratios between 
Planck units. These constants demonstrate that while individual Planck units lie outside the range of direct measurement [13], the ratios between Planck units can be measured accurately.

Each measured ratio between Planck units provides a constraint on the possible values of the Planck units. Applying these constraints collectively through the science of metrology may improve the values of all derived constants.

Under the redefinition of the International System of Units in May 2019, Planck's constant and the speed of light are given exact values while CODATA values of the Planck units have a relative standard uncertainty of $1.1 \times 10^{-5}$. Using the precise values of Planck's constant and the speed of light as expected values, and the Planck unit formulas for $\hbar$ and $c$ in table 2, we can assess the degree to which the Planck units vary from expected values. Table 15 compares the exact values with values calculated using the Planck length, mass, and time. Note that calculations performed using CODATA Planck units $d o$ fall within expected ranges of uncertainty.

Table 15: Deviation between exactly defined constants and values calculated using the Planck units provide information for improving the values of physical constants.

\begin{tabular}{lllll}
\hline Constant & Exact value & Planck formula & Planck unit value & Deviation \\
\hline$c$ & $299,792,458 \mathrm{~m} / \mathrm{s}$ & $l_{P} / t_{P}$ & $299,792,423 \mathrm{~m} / \mathrm{s}$ & 0.99999988 \\
$\hbar$ & $1.054571817 \times 10^{-34} \mathrm{kgm}^{2} / \mathrm{s}$ & $l_{P}^{2} m_{P} / t_{P}$ & $1.05457151 \times 10^{-34} \mathrm{kgm}^{2} / \mathrm{s}$ & 0.99999971 \\
$\hbar / c$ & $3.51767294 \times 10^{-43} \mathrm{kgm}$ & $l_{P} m_{P}$ & $3.51767233 \times 10^{-43} \mathrm{kgm}$ & 0.99999983 \\
$\hbar / \mathrm{c}^{2}$ & $1.17336939 \times 10^{-51} \mathrm{kgs}$ & $m_{P} t_{P}$ & $1.17336933 \times 10^{-51} \mathrm{kgs}$ & 0.99999995 \\
\hline
\end{tabular}

From the table we see that the product of Planck mass and time is closest to its target value, while the ratio $l_{P}^{2} m_{P} / t_{P}$ is furthest.

We can also use the symmetries and equations presented in section 3 to evaluate the accuracy of measured values compared to theoretical values. For example, 10 requires that each value in table 16 be the same (shown in non-reduced form).

Table 16: Proposed symmetries offer theoretical constraints on the value of constants. According to the New Foundations Model, each of the following values should be the same.

\begin{tabular}{ll}
\hline Formula & Value \\
\hline$h / c$ & $2.2102190943 \times 10^{-42}$ \\
$\lambda_{C} m_{e}$ & $2.2102190943 \times 10^{-42}$ \\
$\lambda_{C, \mu} m_{\mu}$ & $2.2102190937 \times 10^{-42}$ \\
$2 \pi l_{P} m_{P}$ & $2.2102187129 \times 10^{-42}$ \\
\hline
\end{tabular}

The ratio of $h / c$ in the first row is exactly defined under the 2019 redefinition of units. Rows two and three show values of the same constant calculated using the measured mass and Compton wavelength of the electron and muon. Row four calculates the quantum constant using current values of Planck length and Planck mass. Again, the values fall within CODATA ranges of uncertainty, but the comparison shows how we can use different measured values and formulas to calibrate the constants. This example shows the degree to which the value of $l_{P} m_{P}$ differs from values produced using measurements of the electron 
and muon, as well as measurements of Planck's constant and the speed of light. We also learn how much the product of Planck length and mass needs to increase to meet the defined, target value.

Table 15 shows that Planck's constant and the speed of light yield accurate values for three out of the six product and quotient relationships between pairs of fundamental Planck units (where the precision of $\hbar$ and $c$ constitute accurate values). We get accurate values for two of the three product relationships in $l_{P} m_{P}$ and $m_{P} t_{P}$, but no value for $l_{P} t_{P}$. We get an accurate value for one of the three quotient relationships in the speed of light (length and time), but no value for the quotients of length and mass, or time and mass.

Given current information, an accurate measurement of any one of the three remaining paired relationships enables the derivation of all three extensive Planck unit values. For example, an accurate value of $l_{P} t_{P}$ can be used to isolate $m_{P}^{2}$ in the formula $l_{P} t_{P} m_{P}^{2}\left(\hbar^{2} / c^{3}\right)$. With Planck mass we can determine Planck length using the quantum constant $l_{P} m_{P}$, and the value of $t_{P}$ from the speed of light, $l_{P} / t_{P}$.

Similarly, it is easy to show that quotients of either mass and time or mass and length yield values of all three natural units.

In section 4, I showed that ratios of length and mass to their Planck unit values determine the strength of massive bodies on the gravitational field, where the quotient of Planck length and mass is embedded in the gravitational constant. The remaining component of the gravitational constant, $c^{2}$, is well defined, suggesting that an accurate measurement of $l_{P} / m_{P}$ will improve the value of the gravitational constant. In fact, the pursuit of a more accurate gravitational constant can be superseded by the objective of producing a more accurate value of $l_{P} / m_{P}$ as this not only improves $G$ but also improves the values of all constants comprised of the fundamental Planck units. In addition, equation 2 and section 4 depict the gravitational constant as an artifact constructed from more elemental units of length, mass, and time.

Obtaining a more precise measurement of the gravitational constant has proved difficult in an earth environment. The inhomogeneous distribution of matter makes it difficult to reproduce similar measurement results over different times and places. Redefining the objective as measuring $l_{P} / m_{P}$ may reveal alternatives to current measurement methodologies.

Given the new Planck unit formulas in table 2, it is possible to improve the value of the traditional gravitational constant in indirect ways. While any measurement, constraint, or theoretical insight that improves the value of $l_{P} / m_{P}$ improves the value of $G$, an accurate measurement of $l_{P} t_{P}$ or $m_{P} / t_{P}$ will also produce accurate values of all three Planck units and all the constants comprised of them, including $G$. The pursuit of a more accurate gravitational constant can therefore expand its scope to the measurement of these two pairs.

Although Planck's constant and the speed of light do not provide enough information to derive accurate values of the fundamental Planck units, they provide sufficient information to create consistent sets of Planck units. For a given value of one unit, values of the other two units can be calculated in the proper proportions defined by $\hbar$ and $c$, using the formulas in table 15.

Figure 10 illustrates the collective inconsistency of current CODATA Planck unit values, given the ratios defined by Planck's constant and the speed of light. Each node of the equilateral triangle represents one of the three fundamental Planck units with current CODATA values indexed at 1.0. Three proportionally consistent sets of Planck units are plotted as triangles above the base triangle, where one node of each triangle is the indexed CODATA value and the other two Planck units are calculated using the formulas in table 15. For example, the blue triangle uses the CODATA value of Planck length, $1.616255 \times 10^{-35} \mathrm{~m}$, and plots corresponding values of Planck mass and time in the correct proportions. Each proportion- 


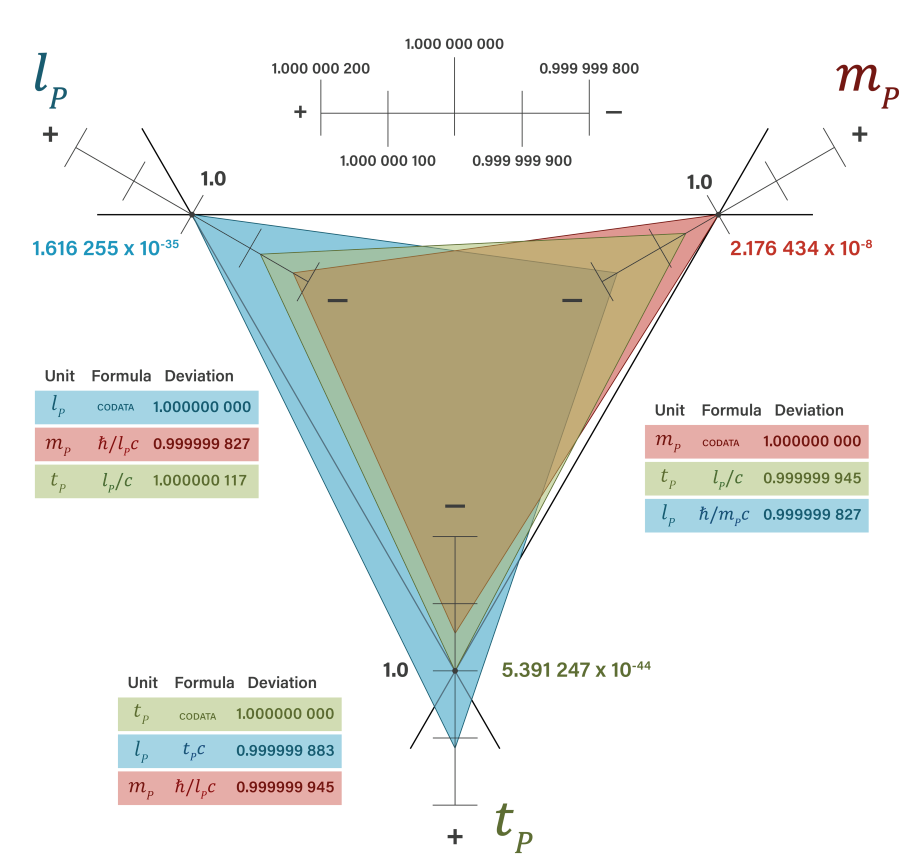

Fig. 10: caption

ally consistent set of Planck units produces defined values of $\hbar$ and $c$ exactly. Proportionally consistent values of the Planck units fall within CODATA ranges of uncertainty.

\section{A Natural Unit System}

The New Foundations Model defines a natural unit system based on two key assumptions. The first assumption is that the fundamental Planck units of length, mass, and time have definitive quantities coinciding at a point of maximum potential. The second assumption is that all three unit types have the same quantized intervals of length, making proportions comparable across unit types. According to this system, the three fundamental Planck units can be described as follows

- Planck Length The Planck length is the minimum natural unit of length. Quantities of length are integer multiples of the Planck length. Length potential has a maximum value of 1 at the Planck length and approaches 0 at infinite distance.

- Planck Mass The Planck Mass is the maximum natural unit of mass and the greatest mass potential. A particle's rest mass reduces its maximum potentials from the Planck scale down to the Compton scale. Mass potential has a maximum value of 1 at the Planck length and approaches 0 at infinite distance.

- Planck Time The Planck time is the minimum natural unit of time. Time is quantified in integer units of the Planck length, where one unit of time is equal to one unit of length counted at the speed of light. Time potential has a maximum value of 1 at the Planck length. 
The natural unit system works by combining the three fundamental units into different ratios, producing maximum potentials of natural phenomena. Table 2 summarizes the maximum potentials and operators embedded in most of the historical constants; however, the traditional form of these constants is no longer necessary. With the knowledge of how these constants function, it is easier to construct maximum potentials and operators specifically for a given question.

The practice of assigning constants like $c, \hbar$ and $G$ a value of 1 follows naturally from the principle of maximum potential and proportionality. Different unit types are normalized when maximum potentials are set to 1 and proportionality operators are constructed on a scale of 0 to 1 . The New Foundations Model clarifies the natural unit scale including how to assign these values.

\section{Conclusion}

More than 120 years after Max Planck proposed natural units of length, mass, and time, the constants used predominantly in physics today are composite constants including $\hbar$ and $G$. The physical meaning of these constants is hidden in the ambiguity of their values. The composite quantities fail to explain why Planck's constant gives the momentum and energy of a photon, or why the gravitational constant determines the force generated by a massive body. Replacing these constants with natural units of length, mass, and time offers a refreshing look at the physical dynamics encoded in the equations of physics.

The New Foundations Model of physics is the first attempt at incorporating this new information into a physical description of the world. It challenges the presumed incompatibility between an intuitive description of the quantum universe, and the abstract mathematical formulations developed over the past century. While it doesn't address the genuinely, nonclassical phenomenon of wave function collapse, it lifts the veil over many abstruse physical transformations revealing quantities of length, mass, and time. It justifies the consideration of quantum oscillations as more than mathematical symbols, but as veritable, physical dynamics.

The model is appealing because of its simplicity in explaining natural laws. It reduces the long inventory of composite constants down to three fundamental units, and replaces at least nine electromagnetic units with units of length, mass, and time. From two equalities it defines momentum, velocity, acceleration, force, action, and energy in the simple, conserved relationships between the three fundamental units.

The model's applicability to particle mechanics, gravity, and electromagnetism portends a deeper unification in our understanding of the natural universe and may contribute to the search for a grand unified theory. The principles of maximum potential and proportionality shed new light on existing theories and will catalyze interesting new theories of the natural universe.

The New Foundations Model will have a positive impact the field of metrology. Countless experiments have steadily improved the accuracy of physical constants that can now be interpreted as ratios between natural units of length, mass, and time. Incorporating this new model into measurement sciences will improve the Planck unit values as well as the constants they define.

The question may be asked why it has taken so long to recognize the importance of natural units in the construct of physical constants, and to incorporate this information into a description of the natural world. Contemporary theories are deeply abstract, and until the mathematical formulations invoke a better understanding of the universe in physically 
meaningful ways, these theories will remain on tenuous foundations. Indications that our understanding is improving will become apparent as fewer aspects of theories become subject to interpretation, as more theories are ruled out, and as consensus grows within the scientific community on the meaning of preferred theories.

The imperative that physical theories offer more than a mathematical formulation in their ability to explain the physical universe was expressed well by David Deutsch

" Being able to predict things or to describe them, however accurately, is not at all the same thing as understanding them...Facts cannot be understood just by being summarized in a formula, any more than by being listed on paper or committed to memory. They can be understood only by being explained." ( [19])

\section{A Derivations}

The principle of maximum potential gives a simple method for determining the Planck unit formulas of composite constants - the formulas match the dimensionality of the constant. Formulas are easily confirmed with calculation.

Formulas for the gravitational constant and Planck's constant can also be derived from the traditional formulas of Planck length and mass. The two formulas stated in terms of the gravitational constant are

$$
G=\frac{l_{P}^{2} c^{3}}{\hbar}
$$

and

$$
G=\frac{\hbar c}{m_{P}^{2}}
$$

Setting the equations equal to each other yields a formula for $\hbar$ in terms of Planck length, Planck mass, and the speed of light

$$
\frac{l_{P}^{2} c^{3}}{\hbar}=\frac{\hbar c}{m_{P}^{2}} .
$$

Simplifying gives equation 1. The equations for Planck length and Planck mass can also be written in terms of Planck's constant

$$
\hbar=\frac{l_{P}^{2} c^{3}}{G}
$$

and

$$
\hbar=\frac{G m_{P}^{2}}{c} .
$$

Setting them equal to each other yields a formula for the gravitational constant in terms of Planck length, Planck mass, and the speed of light

$$
\frac{l_{P}^{2} c^{3}}{G}=\frac{G m_{P}^{2}}{c}
$$

Simplifying gives equation 2 .

The Planck charge is a maximum potential that determines the values of a number of other constants. The Planck charge can be derived using equations for Planck inductance that appeared on the Planck units Wikipedia page in November 2019

$$
L_{P}=\frac{E_{P}}{I_{P}}=\frac{m_{P} l_{P}^{2}}{q_{P}^{2}} .
$$

Restating this equality in terms of the Planck current gives

$$
I_{P}=\frac{E_{P} q_{P}^{2}}{m_{P} l_{P}^{2}} .
$$


Substituting the equation for Planck current into the equation for Planck charge

$$
q_{P}=I_{P} t_{P}
$$

produces an equation for the Planck charge in terms of the MKS units

$$
q_{P}=\frac{E_{P} q_{P}^{2} t_{P}}{m_{P} l_{P}^{2}} .
$$

Reducing the equation gives

$$
q_{P}=\frac{m_{P} l_{P}^{2} t_{P}^{2}}{m_{P} l_{P}^{2} t_{P}}=t_{P} .
$$

Evidence that the Planck charge is equal to the Planck time is found in the consistency of the electromagnetic units given in table 12, the formulas given in table 2, and in the results of calculations performed using formulas like Coulomb's law when charge is converted to seconds.

\section{References}

1. M. Planck, Sitzungsberichte der Königlich Preußischen Akademie der Wissenschaften zu Berlin 5(1), 440 (1899)

2. G. Gamow, D. Ivanenko, L. Landau, Physics of Atomic Nuclei 65(7), 1373 (2002)

3. J.D. Barrow. From alpha to omega, the constant of nature (2003)

4. B.K. Parida, Science Horizon p. 4 (2019)

5. J.R. Buczyna, C. Unnikrishnan, G.T. Gillies, Gravitation and Cosmology 17(4), 339 (2011)

6. E. Gaarder Haug, Physics Essays 29(4), 558 (2016)

7. D. Di Mario, Journal of Theoretics 5(1), 5 (2003)

8. A. Lehto, Nonlinear Dynamics 55(3), 279 (2009)

9. K. Tomilin, (1998)

10. F. Cooperstock, V. Faraoni, International Journal of Modern Physics D 12(09), 1657 (2003)

11. P.J. Mohr, D.B. Newell, B.N. Taylor, REVIEWS OF MODERN PHYSICS Rev Mod Phys 88(3), 035009 (2016)

12. J.R. Buzcyna, C. Unnikrishnan, G.T. Gillies, Grav. Cosmol. 17(arXiv: 1006.3241), 339 (2010)

13. R.J. Adler, American Journal of Physics 78(9), 925 (2010)

14. C.J. Bordé, Philosophical Transactions of the Royal Society A: Mathematical, Physical and Engineering Sciences 363(1834), 2177 (2005)

15. E. Haug, (2018)

16. R.J. Adler, D.I. Santiago, Modern Physics Letters A 14(20), 1371 (1999)

17. N. Bohr, The London, Edinburgh, and Dublin Philosophical Magazine and Journal of Science 26(153), 476 (1913)

18. D. Hestenes, Foundations of Physics 20(10), 1213 (1990)

19. D. Deutsch, The fabric of reality (Penguin UK, 1998) 